NATIONAL LABORATORY

\title{
Spatial Distribution of Induced Fission from the Pixilated Alpha Detector in a DT Generator
}

February 2008

Prepared by Steven Frederiksen John T. Mihalczo

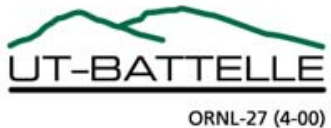




\section{DOCUMENT AVAILABILITY}

Reports produced after January 1, 1996, are generally available free via the U.S. Department of Energy (DOE) Information Bridge.

Web site http://www.osti.gov/bridge

Reports produced before January 1, 1996, may be purchased by members of the public from the following source.

National Technical Information Service

5285 Port Royal Road

Springfield, VA 22161

Telephone 703-605-6000 (1-800-553-6847)

TDD 703-487-4639

Fax 703-605-6900

E-mail info@ ntis.gov

Web site http://www.ntis.gov/support/ordernowabout.htm

Reports are available to DOE employees, DOE contractors, Energy Technology Data Exchange (ETDE) representatives, and International Nuclear Information System (INIS) representatives from the following source.

Office of Scientific and Technical Information

P.O. Box 62

Oak Ridge, TN 37831

Telephone 865-576-8401

Fax 865-576-5728

E-mail reports@osti.gov

Web site http://www.osti.gov/contact.html

This report was prepared as an account of work sponsored by an agency of the United States Government. Neither the United States Government nor any agency thereof, nor any of their employees, makes any warranty, express or implied, or assumes any legal liability or responsibility for the accuracy, completeness, or usefulness of any information, apparatus, product, or process disclosed, or represents that its use would not infringe privately owned rights. Reference herein to any specific commercial product, process, or service by trade name, trademark, manufacturer, or otherwise, does not necessarily constitute or imply its endorsement, recommendation, or favoring by the United States Government or any agency thereof. The views and opinions of authors expressed herein do not necessarily state or reflect those of the United States Government or any agency thereof. 


\title{
SPATIAL DISTRIBUTION OF INDUCED FISSION FROM THE PIXILATED ALPHA DETECTOR IN A D-T GENERATOR
}

\author{
John T. Mihalczo
}

Date Published: February 2008

\author{
Prepared by \\ OAK RIDGE NATIONAL LABORATORY \\ Oak Ridge, Tennessee 37831-6283 \\ managed by \\ UT-BATTELLE, LLC \\ for the \\ U.S. DEPARTMENT OF ENERGY \\ under contract DE-AC05-00OR22725
}





\section{CONTENTS}

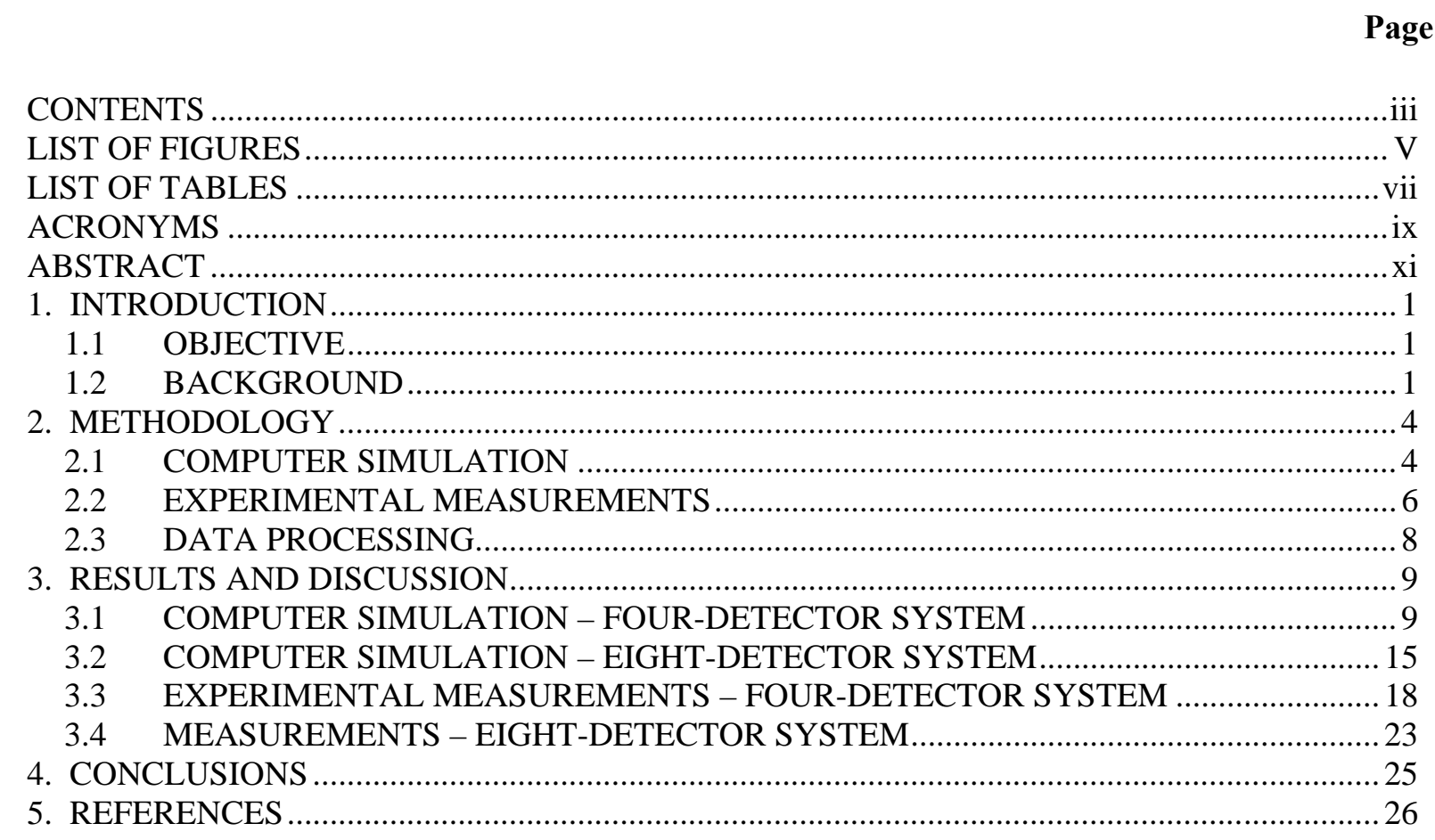





\section{LIST OF FIGURES}

Figure

Page

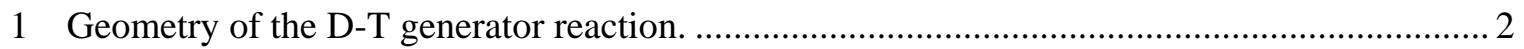

2 Depiction of MCNP-PoliMi model with four detectors. .......................................................... 5

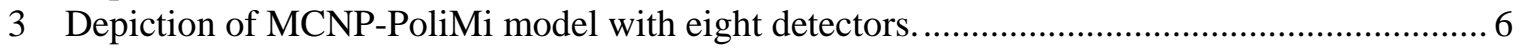

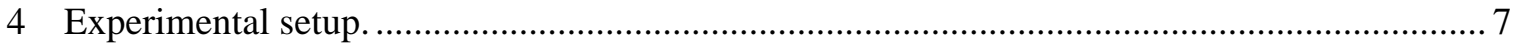

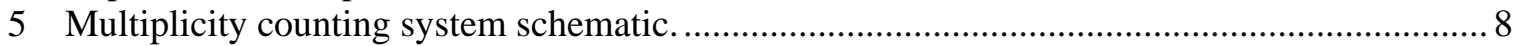

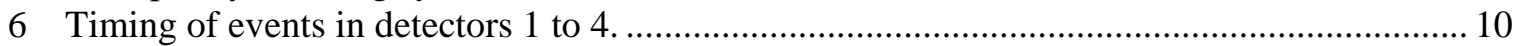

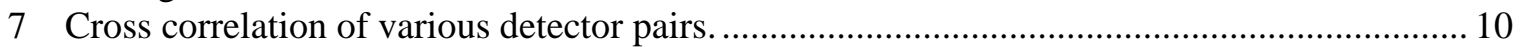

8 Feynman variance by pixel from simulation results for a 16 to 27 ns time window

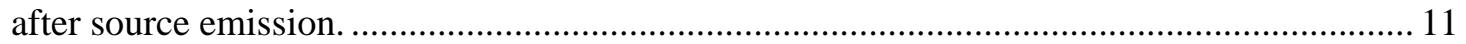

9 Feynman variance by pixel from simulation results for various time windows after

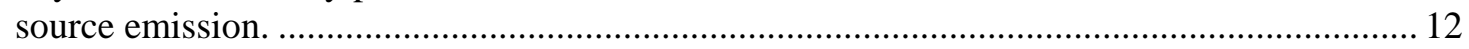

10 Probability that $n$ pulses occur in the 20 to 40 ns window for pixels 01 and $05 \ldots \ldots \ldots \ldots \ldots \ldots . . . . . . .12$

11 PoliMi simulation results for DU annulus with side toward denerator. ................................ 13

12 PoliMi simulation results for DU annulus with opening toward generator............................ 13

13 PoliMi simulation results for two annuli separated by polyethylene. .................................. 14

14 Timing of the detection in the DU-polyethylene-DU configuration................................... 14

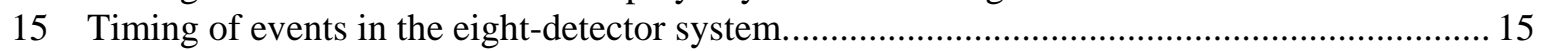

16 Cross correlation of various detector pairs. .......................................................................... 15

17 Feynman variance by pixel for the eight-detector simulation for various time

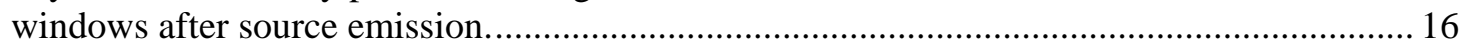

18 PoliMi simulation results for DU annulus with side toward generator. .................................. 17

19 PoliMi simulation results for DU annulus with opening toward generator........................... 17

20 PoliMi simulation results for two annuli separated by water. .............................................. 18

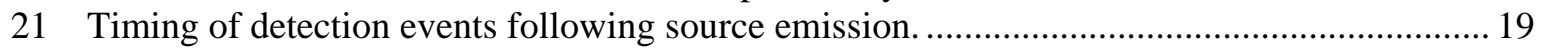

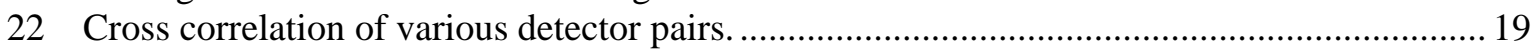

23 Feynman variance by pixel from experimental results for various time windows

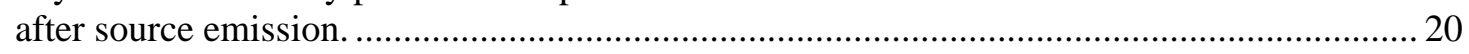

24 Probability that $n$ pulses occur in the 18 to 40 ns window for pixels 01 and $07 \ldots \ldots \ldots \ldots \ldots \ldots . . . . . .21$

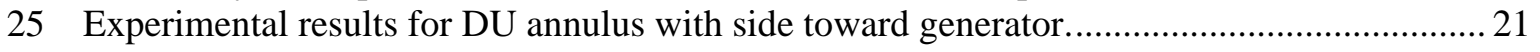

26 Experimental results for DU annulus with opening toward generator. ................................... 22

27 PoliMi simulation results for two annuli separated by polyethylene. ….................................. 22

28 Timing of the detection in the DU-polyethylene-DU configuration.................................... 23

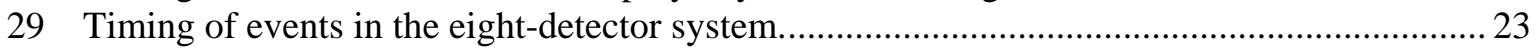

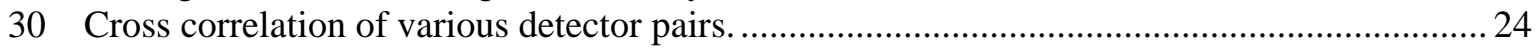

31 Feynman variance by pixel for the eight-detector measurements for various time

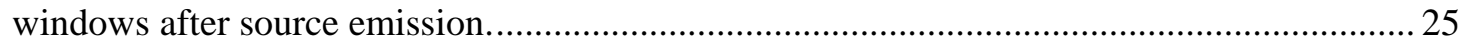

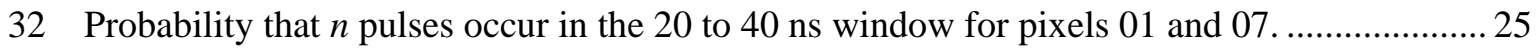





\section{LIST OF TABLES}

Table

Page

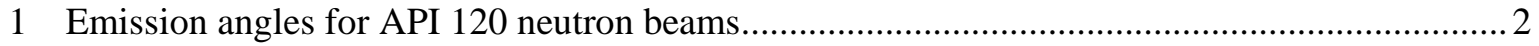

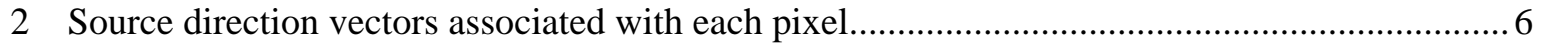

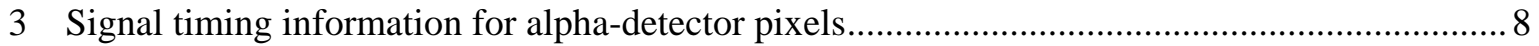

4 Time windows investigated for the four-detector PoliMi simulation...................................... 10

5 Time windows investigated for the eight-detector PoliMi simulation .................................... 16

6 Time windows investigated for the four-detector experimental measurements ........................ 20

7 Time windows investigated for the eight-detector experimental system ................................. 24 



\section{ACRONYMS}

$\begin{array}{ll}\text { CFD } & \text { constant-fraction discriminator } \\ \text { DAUI } & \text { Data Acquisition and User Interface } \\ \text { D-T } & \text { deuterium-tritium } \\ \text { DU } & \text { depleted uranium } \\ \text { HEU } & \text { highly enriched uranium } \\ \text { MCNP } & \text { Monte Carlo N-Particle } \\ \text { NMIS } & \text { Nuclear Materials Imaging System }\end{array}$





\begin{abstract}
Correlating in time the response of fast organic scintillation detectors located around an object interrogated by neutrons with the events in a pixilated alpha detector embedded in a D-T neutron generator was investigated. These correlated signals will allow localizing of the position of fissile material in the interrogated object. The pixilated alpha detector had eight horizontal sections that subdivided a horizontal fan beam of neutrons into eight slightly overlapping cones, some of which were incident on a depleted uranium metal annular casting and other materials. The deviation of the multiple correlated events from a Poisson distribution and the Feynman variance of detection events that occur within a set time window was used as an indication of the occurrence of fission. The ability to correlate detector response from particles from induced fission with the various pixels of the alpha detector was shown to reveal information about the spatial distribution of induced fission in the target. These Nuclear Material Imaging System active interrogation measurements with depleted uranium were the first experimental tests of the feasibility of this technique.
\end{abstract}





\section{INTRODUCTION}

\subsection{OBJECTIVE}

The purpose of this project was to determine the potential of using the Nuclear Material Imaging System (NMIS) to measure the difference between fissile and nonfissile materials through double, triple, and higher coincidence counting of induced prompt fission neutrons and gamma rays. Current systems treat multiplicity as a bulk property of an object; however, the fast coincidence counting coupled with the NMIS can provide more detailed information as to where fissions occur. This approach will give the NMIS the ability to create a fission density image of an interrogated object.

The Oak Ridge National Laboratory Nuclear Science and Technology Division developed the NMIS, which currently creates an image of an object based on neutron and gamma transmission through the object without providing information regarding the fissile nature of the object. For imaging, the detectors are small for spatial resolution and transmission is used. For obtaining information about induced fission, which is a much smaller signal than the transmission, larger detectors are needed. It is often necessary to determine what a closed container holds to verify the configuration of nuclear weapons components, authenticate the presence or absence of a warhead, track weapons components during the dismantlement process, or determine unknown fissile material configurations for criticality safety and other applications.

\subsection{BACKGROUND}

The NMIS currently interrogates objects using $14.1 \mathrm{MeV}$ neutrons from an API 120 neutron generator. The API 120 generates neutrons through the $t(d, n) \alpha$ reaction. In this reaction, deuterons are accelerated into a tritium-containing target. The alpha particle in this reaction is known as the associated particle to the neutron because it and the neutron are emitted "back to back" in the centerof-mass system. Fig. 1 depicts the reaction in the laboratory system and shows that the two particles will be offset by $4.3^{\circ}$ from the $180^{\circ}$ center-of-mass system due to the momentum of the deuteron. The API 120 has an internally mounted yttrium aluminum perovskite (YAP, YAlO ${ }_{3}$ ) scintillator coupled to a photomultiplier with eight $6 \mathrm{~mm} \times 6 \mathrm{~mm}$ pixels (labeled 01, 03, 05, 07, 09, 11, 13, and 15). This detector is designed to detect the alpha particles associated with neutron emissions in the generator target. By noting the location of the alpha particle detection, information regarding the direction of neutron emission can be determined. The alpha detection also serves as the initiating event for timing of correlated events in other detectors ${ }^{1}$. 


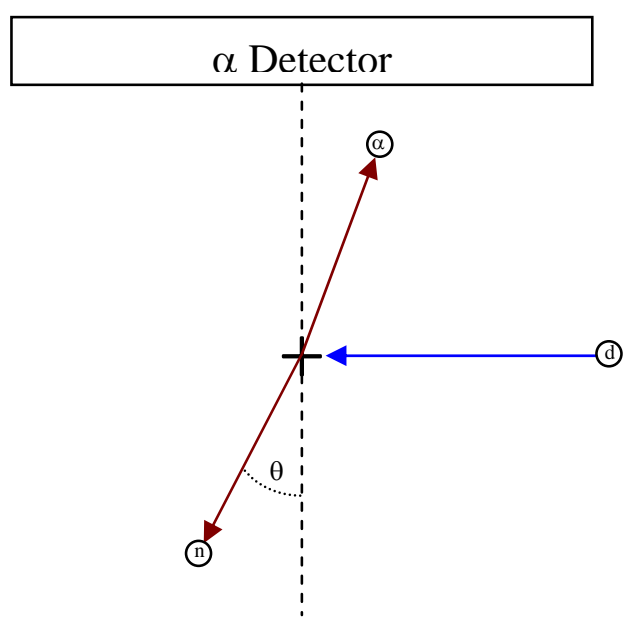

Fig. 1. Geometry of the D-T generator reaction.

An alpha detection in each pixel is associated with a neutron beam emitted from the generator in an approximate $5.5^{\circ}$ cone. Therefore, the eight alpha-detector pixels are associated with a horizontal fan of neutron beams over a $45^{\circ}$ arc. For $90 \mathrm{kV}$ operation of the generator, the neutron fan is offset by about $4.3^{\circ}$ from a line from the center of the pixel array to the center of the generator target because of the momentum of the accelerated deuteron ${ }^{2}$. The neutron beam emission angles $(\theta$, ) are listed in Table 1 . The angles are based off a line extending from the center of the alpha detector to the center of the generator target, as depicted in Fig. 1.

Table 1. Emission angles for API 120 neutron beams

\begin{tabular}{cc}
\hline Pixel & Angle, $\boldsymbol{\theta}\left({ }^{(}\right)$ \\
\hline 01 & 24.2 \\
03 & 18.8 \\
05 & 13.0 \\
07 & 6.8 \\
09 & 1.1 \\
11 & -5.1 \\
13 & -10.8 \\
15 & -16.2 \\
\hline
\end{tabular}

The neutrons emitted from the API 120 interact in an interrogated object through scattering, absorption, and in the case of fissile materials, fission. The NMIS forms a density image of the interrogated object by measuring neutron transmission through the object. The current incarnation of the NMIS detects transmitted neutrons using an array of 1 in. $\times 1$ in. $\times 6$ in. plastic scintillation detectors and correlates these detection events in time with a detection event in the alpha-detector pixels. Induced fission neutrons and gamma rays are not accurately measured with these small detectors $^{3}$.

The use of the NMIS for this project attempted to measure fission interactions within the interrogated object to correlate those measurements with an associated alpha detection. Multiplicity coincidence measurements are based on the assumption that two or more prompt fission particles (neutrons and gamma rays) are emitted nearly simultaneously and instantaneously after absorption of 
the incident neutron and occur on a timescale ten orders of magnitude faster than detector capabilities. In the case of ${ }^{238} \mathrm{U}$ fission initiated by $14.1 \mathrm{MeV}$ neutrons, there are on average 4.4 prompt fission neutrons ${ }^{4}$. Fission neutrons that leave the interrogated object can then be detected in neutron detectors placed around the interrogated object. Neutrons that are released in the same fission event will arrive at the detectors within a time window that is dependent on the geometry of the system.

In the investigation described, in which a fissile object was being interrogated, the average number of counting events, $\bar{c}$, that occurred within a given time window would tend to be higher than if the counting events occurred due to purely random processes. Because purely random fluctuations in the number of counts follow a Poisson distribution, any departure of the probability distribution of the number of counts among the detectors from the Poisson distribution is an indication of nonrandom events ${ }^{5}$. The value in Eq. 1, called the Feynman variance, is a means of measuring this departure.

$$
Y=\frac{\left(\overline{c^{2}}-(\bar{c})^{2}\right)}{\bar{c}}-1
$$

For counting events that occur randomly and follow a Poisson distribution, the Feynman variance will be equal to zero ${ }^{6}$.

To estimate appropriate time windows for detecting fission neutrons, it is necessary to determine the speeds of neutrons emitted in the neutron generator and of neutrons emitted from fissions. Using relativistic mechanics, it can be shown that the relativistic momentum, $p$, of a particle can be given by the expression in Eq. 2.

$$
p=\frac{m_{o} v}{\sqrt{1-\frac{v^{2}}{c^{2}}}}
$$

In this expression, the rest mass of the particle, $m_{o}$, has been adjusted to account for the increase due to the particle's motion. Additionally, it can be shown that the product of the momentum and the speed of light, $p c$, is as given in Eq. 3.

$$
p c=\sqrt{E^{2}-m_{o}^{2} c^{4}}
$$

in which, $E$ is the total energy of the particle. But the energy of the particle is just the sum of the rest mass of the particle and the particle's kinetic energy, $K E$, as shown in Eq. 4.

$$
E=K E+m_{o} c^{2}
$$

Therefore, Eq. 3 can be put in terms of just the kinetic energy of the particle and the particle rest mass, as shown in Eq. 5.

$$
p c=\sqrt{K E^{2}+2 K E m_{o} c^{2}}
$$

In this study, neutrons emitted from the neutron generator had an energy of $14.1 \mathrm{MeV}$, and neutrons emitted during fission had an average energy of $2.3 \mathrm{MeV}^{7}$. Because the rest mass of a neutron is $939.6 \mathrm{MeV} / \mathrm{c}^{2}$, the radical in Eq. 5 is dominated by the second term, so the approximation in Eq. 6 can be made.

$$
p c \approx \sqrt{2 K E m_{o} c^{2}}
$$

Combining Eqs. 2 and 6, the particle speed can be put in terms of the kinetic energy of the particle and its rest mass, as shown in Eq. 7.

$$
v=c \sqrt{\frac{2 K E}{m_{o} c^{2}+2 K E}}
$$

Using Eq. 7, the speed of neutrons emitted from the neutron generator is determined to be 5.1 $\mathrm{cm} / \mathrm{ns}$, and the average speed of fission neutrons is determined to be $2.1 \mathrm{~cm} / \mathrm{ns}$. 
In a system with distances on the order of a meter or less, the time between neutron generation and detection in the surrounding detectors will be on the order of tens of nanoseconds, so fast timing of detection events is necessary. Multiplicity measurements detecting thermal neutrons have previously been conducted using ${ }^{3} \mathrm{He}$ or $\mathrm{BF}_{3}$ gas detectors that detect only thermal neutrons at standard temperature ${ }^{8,9}$. These detectors require numerous scattering interactions during the slowing process, taking many microseconds. Therefore, detection of prompt fission neutrons is spread over many tens of microseconds, and this type of detector is not appropriate for an application in which timing in the scale of nanoseconds is required. For this reason, prompt fission neutrons were detected by an array of LS-301 organic liquid scintillation detectors coupled to high-gain fast photomultiplier tubes. Each detector has a $25 \mathrm{~cm} \times 25 \mathrm{~cm} \times 8 \mathrm{~cm}$ active volume. Previous NMIS multiplicity measurements using fast organic scintillators were performed for passive measurements with plutonium oxide and active measurements at a linac at the Idaho accelerator center ${ }^{10}$.

This project builds on the current capabilities of the NMIS by determining the ability of the system to correlate fission events to a neutron beam associated with a given direction. With this capability, the NMIS will be able to construct not only a material density image of the interrogated object, but also a map of where in the object fissions occur. The neutron transmission cross sections of highly enriched uranium (HEU) and depleted uranium (DU) metals are equal. Thus, transmission imaging cannot distinguish DU from HEU, but the fission density mapping will distinguish HEU from DU. Although 14.1 MeV neutrons induce fission in HEU and DU, the fission-chain multiplication processes in HEU will propagate for longer times and produce higher multiplets. This fact will allow details of the internal design of the object to be discovered. It may be possible to also determine the enrichment of a fissile object from the fission density and the number of neutrons emitted from each fission.

\section{METHODOLOGY}

The research was conducted through both computer simulation and experiment.

\subsection{COMPUTER SIMULATION}

The first model to be constructed and run using the Monte Carlo N-Particle (MCNP) code was to determine the optimum positioning of the liquid scintillator fast neutron detectors. Through analyzing the spatial distribution of fission neutrons exiting a depleted uranium casting, it was determined that the majority of fission neutrons are emitted from the casting in approximately a $120^{\circ}$ cone directed back toward the neutron generator. Ideally then, the liquid scintillator detectors should be placed to the side of the interrogated object with at least some positional bias toward the deuterium-tritium (DT) generator. However, this must be balanced with the fact that detectors located close to the generator have a greater chance of becoming saturated.

The next series of calculations were conducted with the MCNP-PoliMi code to predict the occurrence of double, triple, and quadruple coincidences of neutrons in the detectors with a given alpha-detector pixel. One advantage of the MCNP-PoliMi code over the standard MCNP5 code is that the PoliMi code attempts to closely model the induced fission-chain processes. Standard MCNP models use average numbers of particles emitted in a fission event, which is suitable for modeling on a macroscopic scale. However, when attempting to calculate the time correlation of detection events, it is necessary to have more microscopic detail. The PoliMi code tracks fission processes on an eventby-event basis, and the number of particles emitted in a fission event is determined by sampling from appropriate multiplicity distributions. A second advantage of the PoliMi code is that it allows for more effective modeling of detectors because of its analog tracking of particles through collisions. 
The output of the PoliMi code gives detailed information regarding all collisions occurring in userdefined detector cells to include timing information after the beginning of the history ${ }^{11}$. For the purposes of this investigation, multiple events in a single detector that occurred within $100 \mathrm{~ns}$ after a first event in that detector were not counted. This followed the experimental setup in which the constant-fraction discriminator (CFD) output for each neutron detector had a 100 ns pulse width and therefore a 100 ns dead time.

The general model simulates the experimental setup with a D-T neutron generator, an interrogated DU casting, and four liquid scintillator neutron detectors. The DU casting has an approximate inner diameter of $8.89 \mathrm{~cm}$, an approximate outer diameter of $12.7 \mathrm{~cm}$, and an approximate height of $15 \mathrm{~cm}$. Each detector has an active detection medium consisting of LS-501 liquid scintillator (xylene) in a $25 \times 25 \times 8 \mathrm{~cm}^{3}$ volume. The detector housing consists of a $1 \mathrm{~cm}$ thickness of aluminum, and each detector is shielded against gamma radiation by a $2 \mathrm{~cm}$ thickness of lead on the front face and $1 \mathrm{~cm}$ thickness of lead elsewhere. Fig. 2 is a visualization of the MCNP model. It can be seen that the casting is centered on the origin, the source is located at a distance of 33 $\mathrm{cm}$ from the origin, and the front face of the lead shielding is $19 \mathrm{~cm}$ from the centerline of the system.

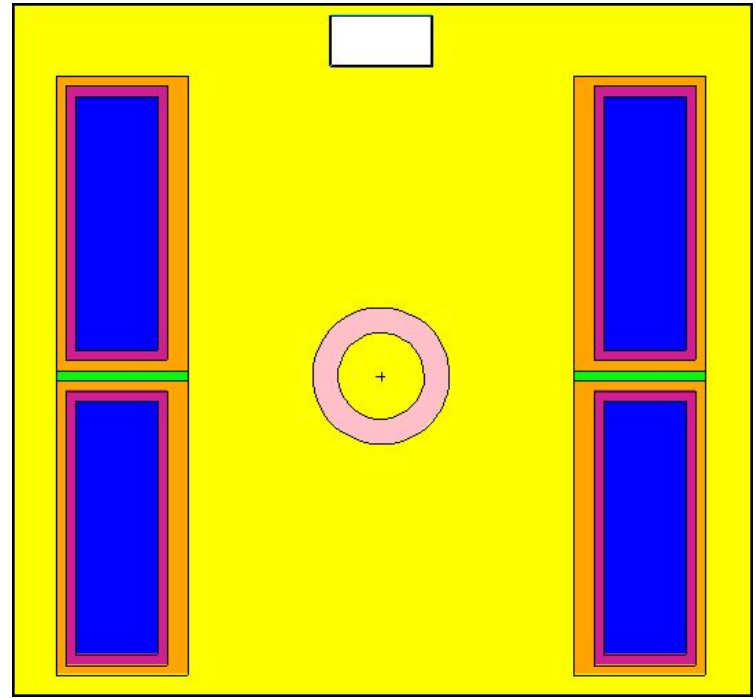

Fig. 2. Depiction of MCNP-PoliMi model with four detectors.

Using the previously determined speeds for neutrons emitted from the generator and neutrons emitted from fission, it is possible to estimate time windows in which different neutron detection events should be expected as a starting point for investigation. In this geometry, neutron beams that are directly incident on the two detectors closest to the generator should be detected between 4 and 9 ns after emission. Those that are directly incident on the two detectors farthest from the neutron generator should be detected between 8 and 13 ns after emission. Prompt fission neutrons from the interrogated casting should arrive at all detectors generally between 16 and $27 \mathrm{~ns}$ after the associated neutron emission in the generator. It is also be useful to check the cross correlation of events in the liquid scintillator detectors to determine the width of the time windows to be investigated.

Eight specific models were developed to simulate the neutron emissions associated with one of the pixels of the neutron generator. Table 2 lists the source-direction information associated with the pixels in the model. As in the actual system, neutrons were emitted in a cone with a width of approximately $4^{\circ}$ degrees, and the beam angles associated with each pixel were offset by about $4^{\circ}$ due to the deuteron momentum. 
Table 2. Source direction vectors associated with each pixel

\begin{tabular}{cccc}
\hline \multirow{2}{*}{ Pixel } & \multicolumn{3}{c}{ Source vector } \\
\cline { 2 - 4 } & $\mathbf{X}$ & $\mathbf{Y}$ & $\mathbf{Z}$ \\
\hline 01 & 0 & 0.45 & -1 \\
03 & 0 & 0.34 & -1 \\
05 & 0 & 0.23 & -1 \\
07 & 0 & 0.12 & -1 \\
09 & 0 & 0.02 & -1 \\
11 & 0 & -0.09 & -1 \\
13 & 0 & -0.19 & -1 \\
15 & 0 & -0.29 & -1 \\
\hline
\end{tabular}

Additional simulations were conducted of an eight-detector configuration, depicted in Fig. 3. The detectors in this configuration were identical to the detectors in the four-detector configuration; however, there was no lead shielding around the detectors themselves. Each detector was separated from adjacent detectors by approximately $15 \mathrm{~cm}$ of polyethylene to reduce the probability of multiple detections of the same neutron. The two banks of detectors were separated by $60 \mathrm{~cm}$, and the source was centered on a point $40.2 \mathrm{~cm}$ from the center of the configuration. Gamma rays from the interrogated object were shielded by a lead annulus with a $1 \mathrm{~cm}$ thickness. In this geometry, prompt fission neutrons from the interrogated casting should arrive at all detectors generally between 25 and 40 ns after the associated neutron emission in the generator.
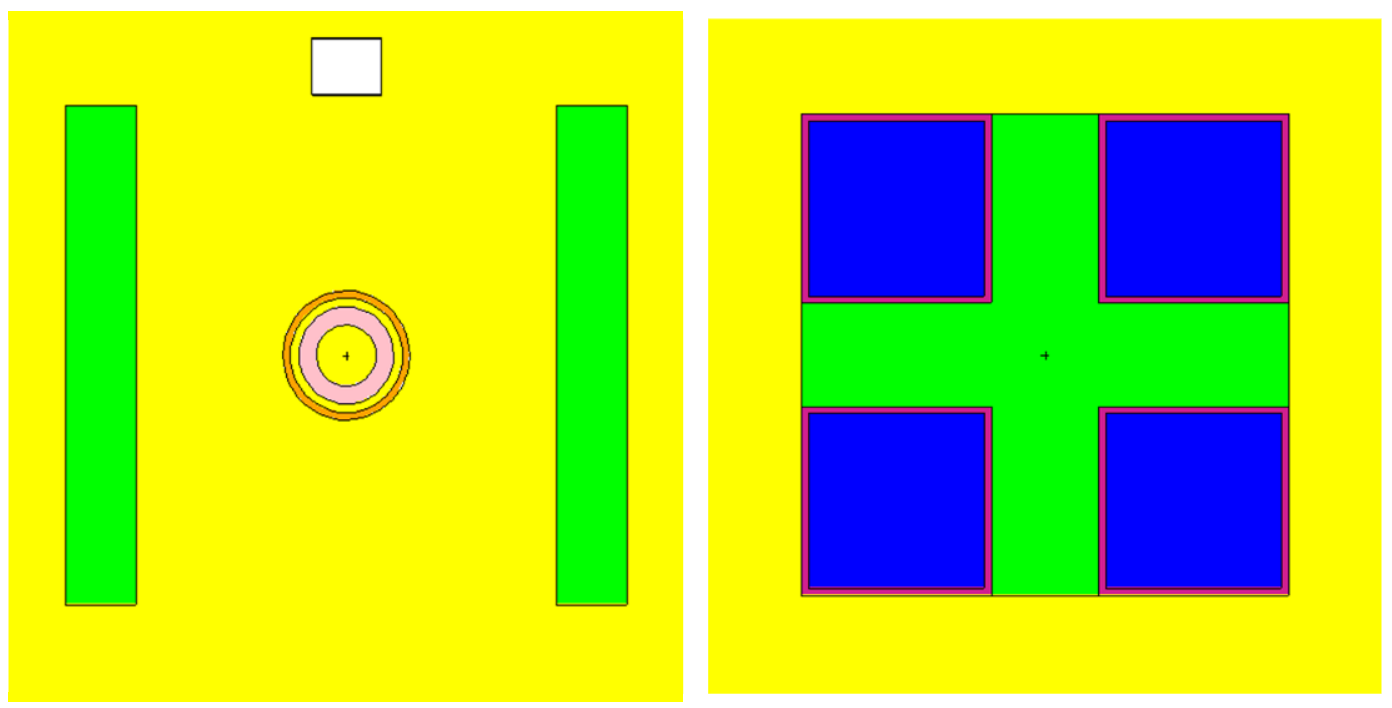

Fig. 3. Depiction of MCNP-PoliMi model with eight detectors.

\subsection{EXPERIMENTAL MEASUREMENTS}

The experimental setup for the multiplicity measurements was designed to closely replicate the system modeled by MCNP-PoliMi. The main difference was that the distance from the source to the center of the DU casting was $44.5 \mathrm{~cm}$ rather than $33 \mathrm{~cm}$. Therefore, only neutrons associated with three of the alpha-detector pixels $(07,09$, and 11$)$ would interact directly with the casting. The pixels were oddly numbered from 1 to 15.The experimental setup is shown in Fig. 4. Tungsten and lead 
blocks were placed between the neutron generator and the liquid scintillators to reduce the number of detection events in the detectors not associated with fissions in the DU. All experimental measurements were made for $3 \times 10^{9} 512 \mathrm{~ns}$ blocks for a total measurement time of 25:36 (min:s).

Because of the geometry differences, the estimated time windows for detection were different than in the simulation. Neutron beams that are directly incident on the two detectors closest to the generator should be detected between 5 and 10 ns after emission. Those that are directly incident on the two detectors farthest from the neutron generator should be detected between 10 and 15 ns after emission. Prompt fission neutrons from the interrogated casting should generally arrive at all detectors between 18 and 29 ns after the associated neutron emission in the generator. Additionally, the cross correlation of events between detectors can be used to determine the width of the time windows to be investigated.

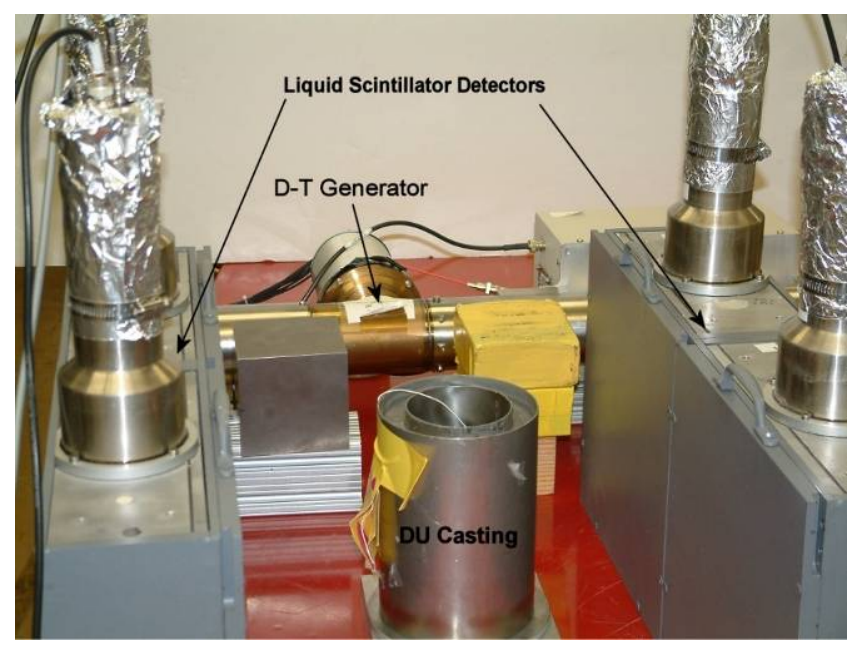

Fig. 4. Experimental setup.

The signal-processing and data-collection counting system is depicted in Fig. 5. All signal processing and detection-count processing were conducted by two NMIS processor boards. Each NMIS processor board had five input channels and gigahertz sampling-rate capabilities ${ }^{3}$. Timing was synchronized between the boards through the use of a single random pulse generator signal. The timing information described in the next paragraphs was programmed into a detector system data file, which allowed the Data Acquisition and User Interface (DAUI) software to collect and record the data through the NMIS processors.

Signals from each organic scintillator were sent through a CFD to shape the signal for timing. Each CFD trigger was set to ensure equivalent detection in each of the detectors. The output pulses from the CFD for each scintillator were set to 100 ns widths, and each detector signal was input into its own channel on an NMIS processor board.

Signals from each of the alpha-detector pixels were sent through a CFD to shape the signal for timing. Each CFD trigger was set to ensure equivalent detection in each of the alpha-detector pixels. Output pulses from the CFD for each pixel were set to allow for multiple pixel signals to be input into a single NMIS processor board channel. Because some alpha-detection events occurred near the edge of pixels, only nonadjacent pixels were input into a single channel. Otherwise, the edge events would be registered in only one pixel. The CFD pulse widths associated with each alpha-detector pixel are listed in Table 3. The CFD outputs for the nonadjacent pixels were combined in a quad linear logic unit, and then the pixel signals were input into two channels in the NMIS processor boards. 


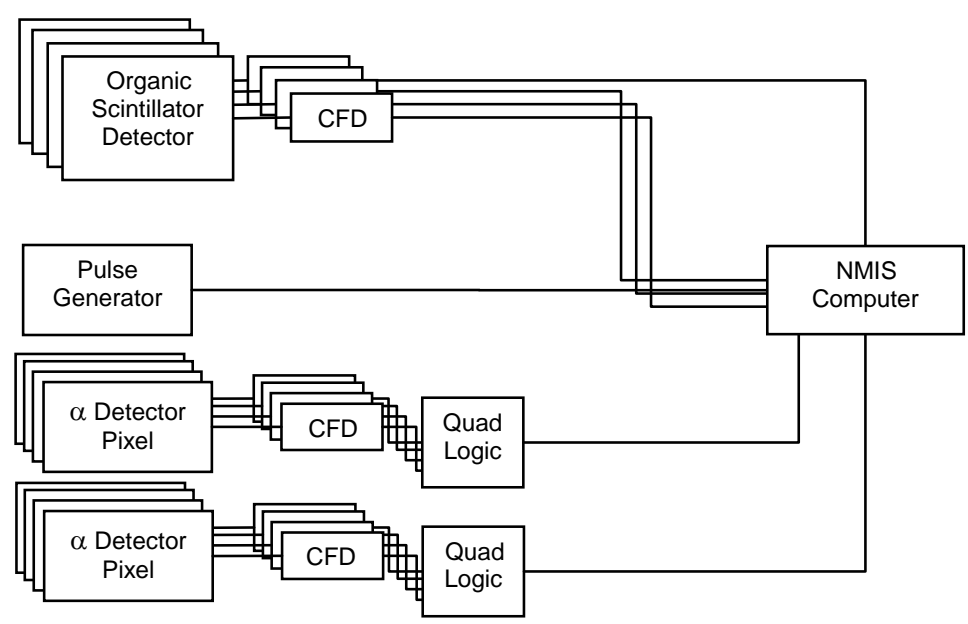

Fig. 5. Multiplicity counting system schematic.

Table 3. Signal timing information for alphadetector pixels

\begin{tabular}{ccc}
\hline Pixel & $\begin{array}{c}\text { CFD output } \\
\text { width (ns) }\end{array}$ & $\begin{array}{c}\text { Quad linear } \\
\text { logic unit }\end{array}$ \\
\hline 01 & 60 & 1 \\
03 & 60 & 2 \\
05 & 80 & 1 \\
07 & 80 & 2 \\
09 & 100 & 1 \\
11 & 100 & 2 \\
13 & 120 & 1 \\
15 & 120 & 2 \\
\hline
\end{tabular}

Prior to conducting the multiplicity measurements, the bias voltages of the liquid scintillator detectors were set so each of the detector responses would be the same for like neutrons. Efficiency measurements were made using DAUI and analyzed with the Interactive Data Analysis Software package. These packages generate efficiency values for organic scintillators using time-of-flight measurements of neutrons emitted from a ${ }^{252} \mathrm{Cf}$ ionization chamber. These measurements were conducted at a distance of $158 \mathrm{~cm}$ from the source, and the bias for each detector was set to achieve $60 \%$ efficiency for the $2.3 \mathrm{MeV}$ neutrons emitted from the source.

Initial measurements were also performed using an eight-detector configuration that matches the simulated eight-detector configuration. The signal processing and data acquisition for the alpha detectors were identical to those for the four-detector configuration. However, due to the limited number of input channels to the NMIS computer, it was necessary to combine the geometricallyopposite detector pairs into one input signal in a manner similar to the way the alpha-detector signals were combined. Because the geometry of this configuration was the same as that in the simulation, prompt fission neutrons were also expected to arrive at the detectors generally 25 to 40 ns after an associated emission in the neutron generator.

\subsection{DATA PROCESSING}

The first step in processing both the PoliMi output and the experimental data was to extract the timing information from the data files to determine the optimum time windows for detecting multiple gamma rays and neutrons from fission. With the timing information, it was possible to approximate 
the time when particles from fission began to be detected in the various detectors surrounding the interrogated object. Further analysis of the timing information included examining the cross correlation between detector pairs to estimate the time window width that would detect the largest number of coincident fission emissions. The time windows to be investigated were then determined from these parameters and the previous time-of-flight calculations.

Multiple time windows were investigated for each configuration. Within each of the evaluated time windows, it was possible determine the probability that multiple events would occur among the detectors. The occurrence of multiple events among the detectors was further correlated to individual neutron beams. All of the time windows were evaluated against their ability to distinguish neutron beams that were incident on a multiplying medium from those that were not.

\section{RESULTS AND DISCUSSION}

\subsection{COMPUTER SIMULATION - FOUR-DETECTOR SYSTEM}

Initial analysis of the PoliMi results concerned the timing of events among the detectors. Fig. 6 shows the timing of events in detectors during the first $100 \mathrm{~ns}$ after a source emission for all four detectors. It can be seen that gammas from fission arrived at the detectors at about 9 ns after source emission. Fission neutrons then arrived at the detectors at about $15 \mathrm{~ns}$, with a peak number of fission neutrons arriving at about $25 \mathrm{~ns}$. It can be seen that in detectors 2 and 4, neutrons arrived directly from the generator at about 10 to $12 \mathrm{~ns}$, so the beginning of the fission neutron arrivals cannot readily be seen; however, the directly incident neutron beam signal fell off significantly by about $20 \mathrm{~ns}$.

Fig. 7 shows the cross correlation in time of events occurring in pairs of detectors across the system. Absent from these plots are structure that would be expected from gamma-gamma coincidences and gamma-neutron coincidences because the lead shielding around the detectors and the attenuation in the uranium metal sharply cut the gamma detection signal in the detectors. Looking at the half width at half maximum, it can be seen that generally events occurred in a second detector within 20 ns of a first event. Correlation plots of detector pairs that included detector 2 were skewed due to the high occurrence of source neutrons that were directly incident on that detector and were not useful in determining time windows to be investigated.

To determine the occurrence of fissions associated with each pixel, the 16 to 27 ns time window as well as other expanded time windows were investigated in which fission neutrons would probably be detected in the organic scintillators. The expanded time windows were chosen because the timing plots in Fig. 6 showed that detection of fission events occurred after about 20 ns, and the cross correlation plots in Fig. 7 showed that events between detectors generally occurred within 20 ns of each other. Table 4 lists the time windows that were investigated for the four-detector PoliMi simulation. 

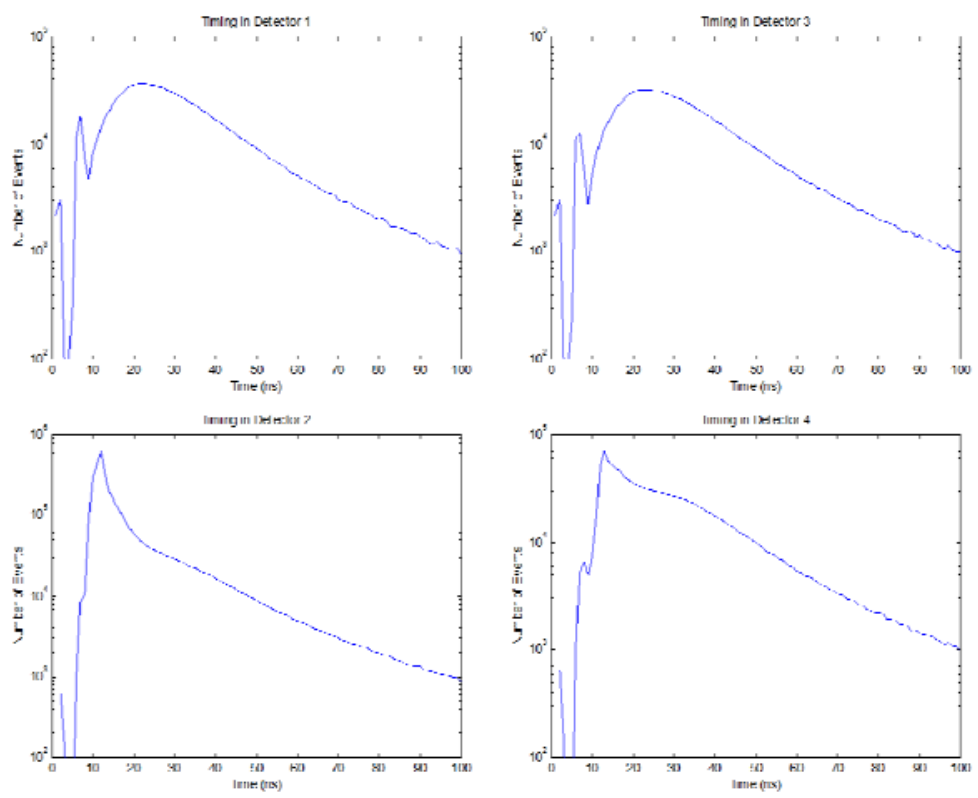

Fig. 6. Timing of events in detectors 1 to 4 .
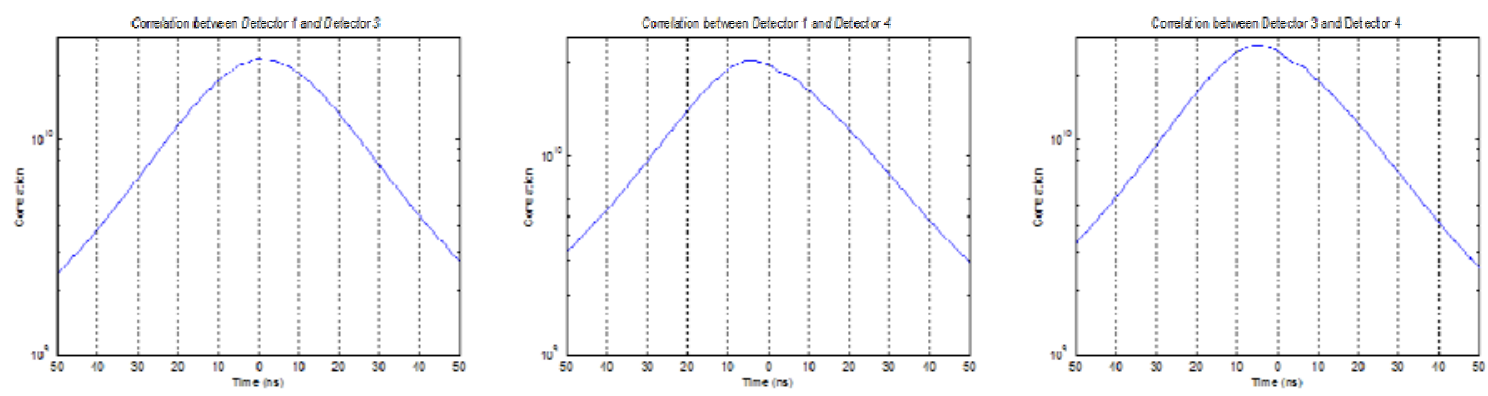

Fig. 7. Cross correlation of various detector pairs.

Table 4. Time windows investigated for the fourdetector PoliMi simulation

\begin{tabular}{c}
\hline Investigated time windows \\
\hline 16 to $27 \mathrm{~ns}$ \\
20 to $30 \mathrm{~ns}$ \\
20 to $35 \mathrm{~ns}$ \\
20 to $40 \mathrm{~ns}$ \\
\hline
\end{tabular}

The Feynman variance by pixel for the 16 to 27 ns time window is shown in Fig. 8. In all subsequent figures, pixels are numbered 1 to 8 instead of as odd numbers between 1 and 15, although they are described in the text by the odd numbers between 1 and 15. Pixels 05 and 13, whose neutron beams were incident on the sides of the casting, had Feynman variances that were significantly above zero. Pixels 07, 09, and 11, whose beams were incident on the center of the casting, had Feynman variance values that were elevated but not significantly above zero. It was expected that the Feynman variance of pixels 01,03 , and 15 would be about zero, but their values were less than zero. This result indicates that there were fewer correlated multiple detections than would randomly occur, probably 
due to overlap of the 16 to 27 ns time window with the time window for neutrons directly incident on the detectors. Therefore, it was necessary to investigate another later time window.

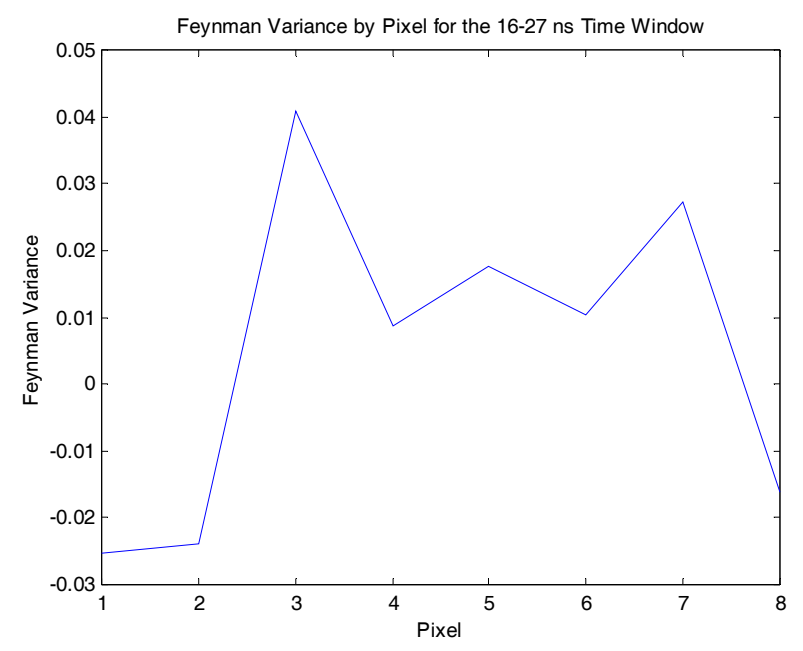

Fig. 8. Feynman variance by pixel from simulation results for a 16 to $27 \mathrm{~ns}$ time window after source emission.

The Feynman variance by pixel for extended time windows is shown in Fig. 9. The values for the Feynman variance were similar to those for the 16 to 27 ns time window for pixels 05, 07, 09, 11, and 13; however, the Feynman variance for pixels 01,03 , and 15 in this time widow were close to zero. This indicates that the center five pixels had more correlated multiple detections than would occur randomly, and their distribution of occurrence should diverge from a Poisson distribution. The three center pixels $(07,09$, and 11) probably had a smaller Feynman variance than the two that had beams incident on the edges of the casting because neutron beams that are incident with the sides of the casting will pass through more material and therefore have a higher macroscopic cross section for fission than the beams in the center. Additionally, neutrons emitted from fissions occurring in the center of are more likely to be absorbed in the casting. This theory can be investigated further through creating simulations that track the progress of prompt neutrons produced in fissions through their entire lifetimes and noting the number that escape from the target. Overall, the 20 to 40 ns time window gave the most evident differences in Feynman variance among the pixels, so it was chosen as the time window to be used in subsequent simulations of different target configurations.

A deviation of the experimental distribution of the number of counts occurring in the 20 to $40 \mathrm{~ns}$ time window from a Poisson distribution for neutron beams incident on the fissile material is consistent with the results depicted in Fig. 9; meanwhile, those beams that are not incident on the fissile material should show little or no deviation from the Poisson distribution. This is further illustrated in Fig. 10, in which the measured distribution of counts closely follows the Poisson distribution for the pixel 01 neutron beam. The pixel 05 neutron beam, being incident on the depleted uranium casting, caused a deviation of the correlated distribution of counts from the Poisson distribution. 


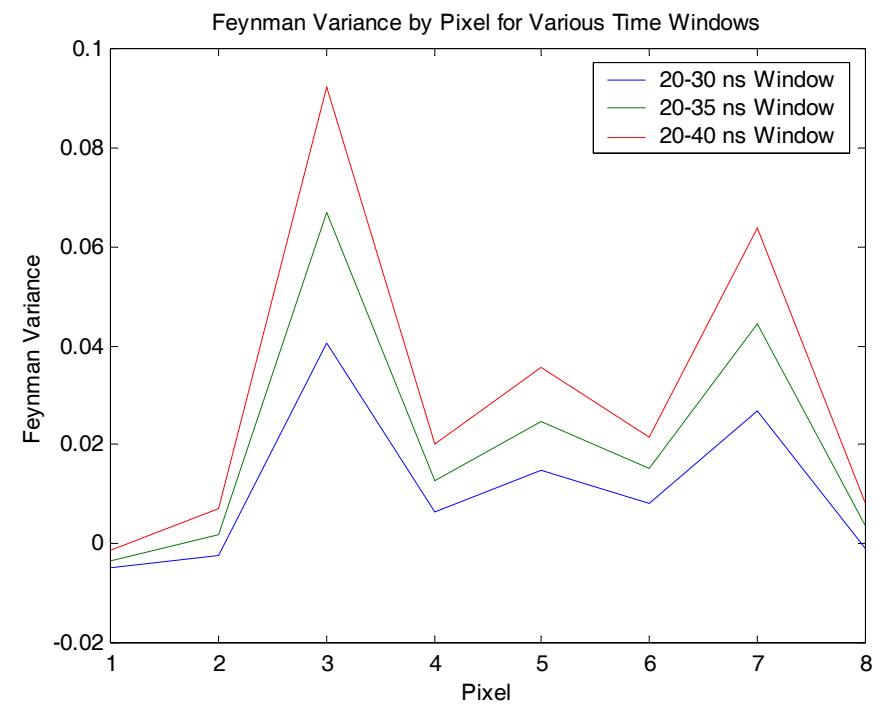

Fig. 9. Feynman variance by pixel from simulation results for various time windows after source emission.
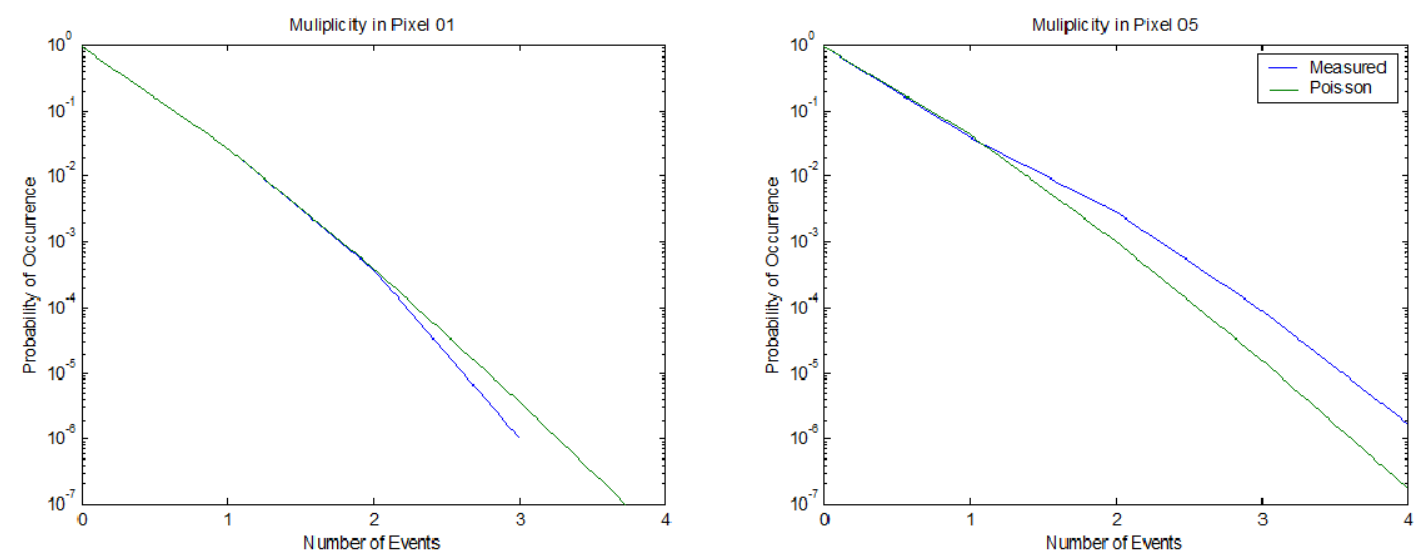

Fig. 10. Probability that $n$ pulses occur in the 20 to 40 ns window for pixels 01 and 05.

Interrogation of DU targets in several different configurations was also simulated with the PoliMi code. The first of these is depicted in Fig. 11, in which the DU annulus has been turned on its side with the side presented to the D-T generator. In this configuration, the pixel 01 neutron beam was not incident on the annulus. The pixel 03 and 15 neutron beams were each partially incident on the annulus. The remaining neutron beams were all directly incident on the target. The Feynman variance plot in Fig. 11 shows elevated values for the pixels with associated neutron beams incident on the annulus, which is consistent with the expected results. 

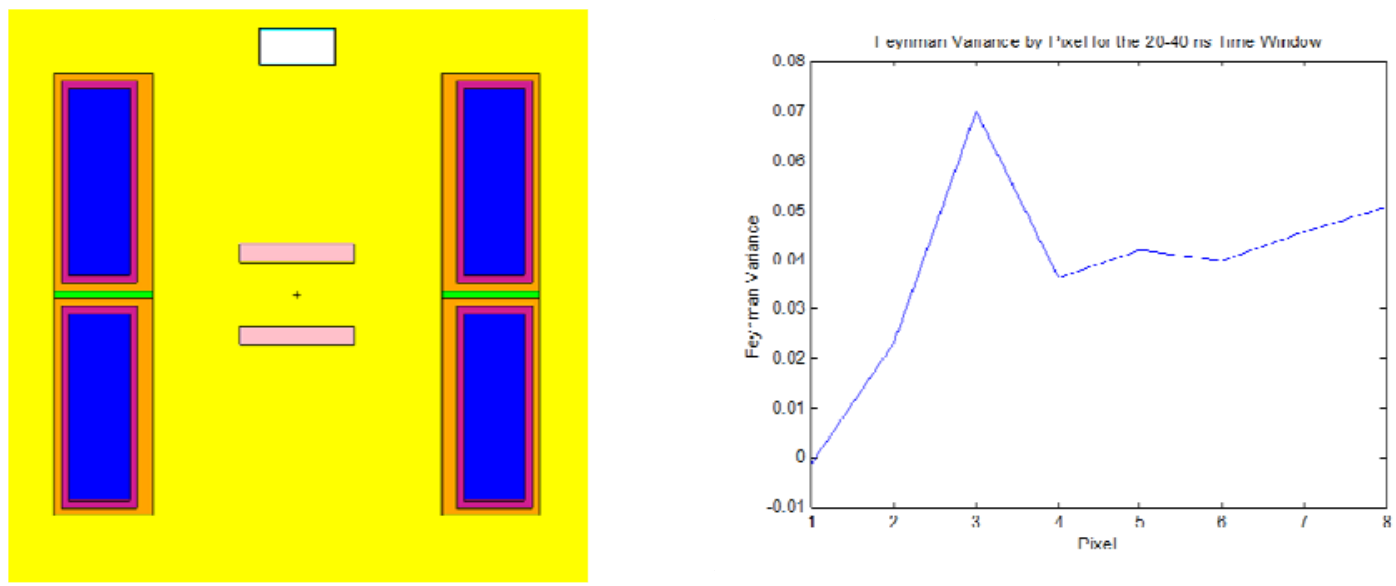

Fig. 11. PoliMi simulation results for DU annulus with side toward denerator.

The next configuration to be simulated was the same DU annulus on its side with the opening presented to the neutron generator, as shown in Fig. 12. In this configuration, neutron beams associated with pixels $05,07,11,13$, and 15 were incident on the leading edge of the annulus. Due to beam spreading, the beam associated with pixel 09 was incident on the inside of the trailing edge of the annulus. The Feynman variance plot in Fig. 12 shows elevated values for the pixels with associated neutron beams incident on the annulus, which is consistent with the expected results.
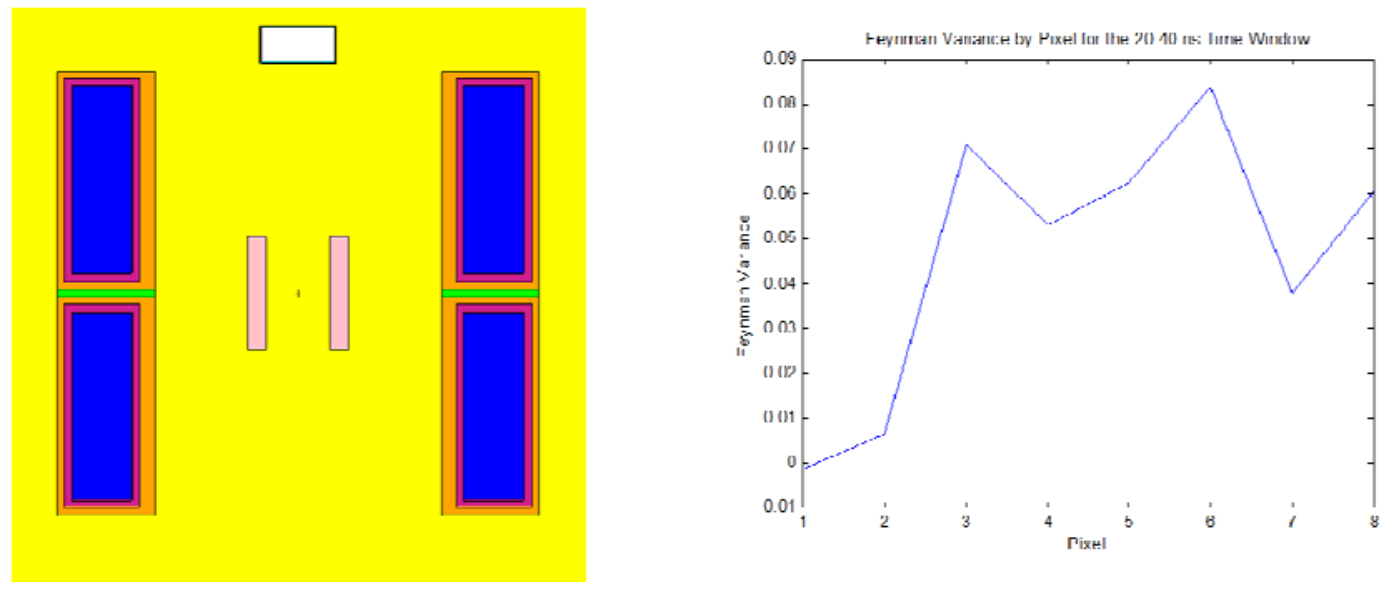

Fig. 12. PoliMi simulation results for DU annulus with opening toward generator.

The final configuration to be simulated with the four-detector system had two DU annuli directly adjacent to the banks of detectors, as depicted in Fig. 13. Both annuli were identical, except the annulus on the right was half the height of the original annulus. The annuli were separated by a cylinder of polyethylene with a height of $6.5 \mathrm{~cm}$, a diameter of $4 \mathrm{~cm}$, and a density of $0.57 \mathrm{~g} / \mathrm{cm}^{3}$. In this configuration, neutron beams associated with pixels $01,03,05,07,13$, and 15 were incident on the DU annuli. The remaining two neutron beams were at least partially incident on the polyethylene cylinder. The Feynman variance plot in Fig. 13 shows several characteristics about this interrogation method. First, the centrally oriented neutron beams that were incident on the polyethylene show elevated Feynman variance values, indicating that neutrons scattered off the polyethylene-induced fissions in the annuli. Additionally, the central 

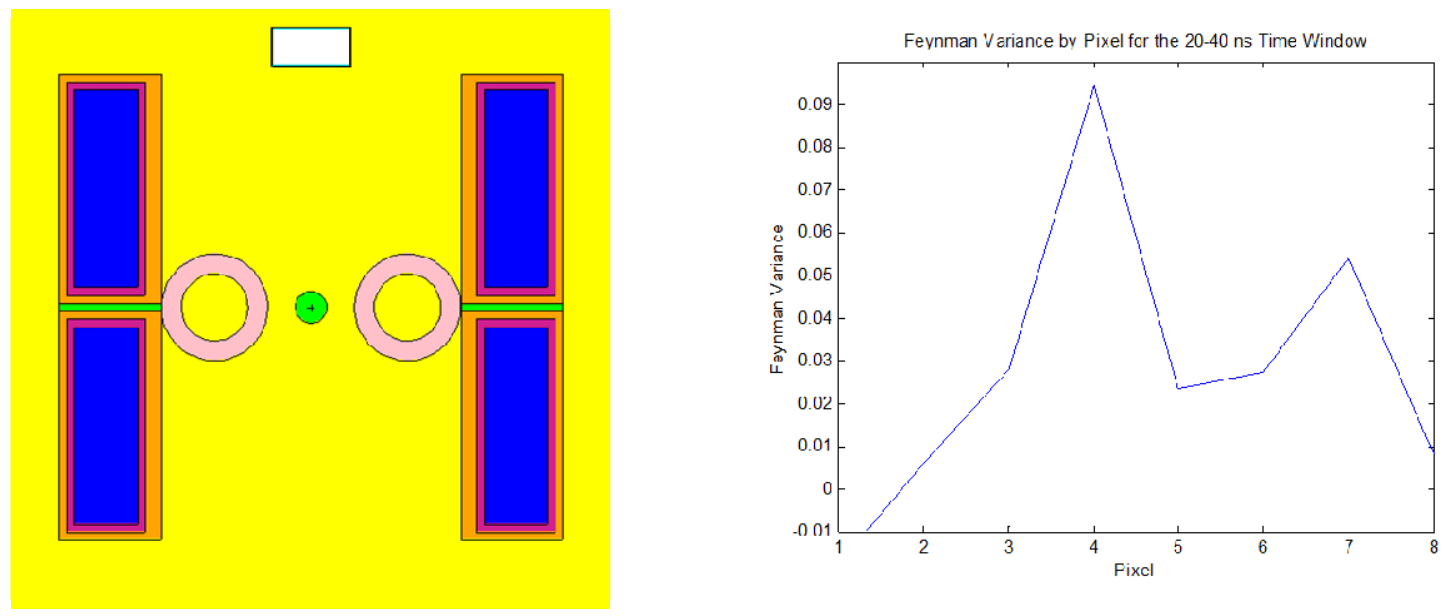

Fig. 13. PoliMi simulation results for two annuli separated by polyethylene.

beams incident on the annuli show different values for the similar locations on the targets. This indicates that more fissions were occurring in the annulus on the left. That was to be expected because the left annulus was larger (15 cm high) and contained more fissile material than the annulus on the right ( $7.5 \mathrm{~cm}$ high).

Finally, the plot shows lower-than-expected Feynman variance values for the beams associated with pixels 01,03 , and 15 . This result can be partially explained by self-attenuation of the targets, and fissions that occur close to one bank of detectors are less likely to produce particles that are detected in the opposite bank of detectors. Therefore, the probability that there will be multiple detections within the investigated time window is greatly decreased because there are effectively only two detectors. The probability of multiple detections in a similar target configuration, therefore, should rise with an increase in the number of detectors in the system.

Additionally, the lower-than-expected Feynman variance values demonstrate the geometric dependency of the selected time window. Due to time of flight of fission particles near the detector banks, detection events should begin at an earlier time and be spread across a wider time window for this configuration. This theory is confirmed by the plots in Fig. 14, in which detection events begin to occur earlier in the detectors of this configuration and peak at an earlier time. If targets are confined, however, to a predetermined volume in the system in which the selected time window has been determined to be valid, then the Feynman variance values should reflect the true multiplicities of the targets.
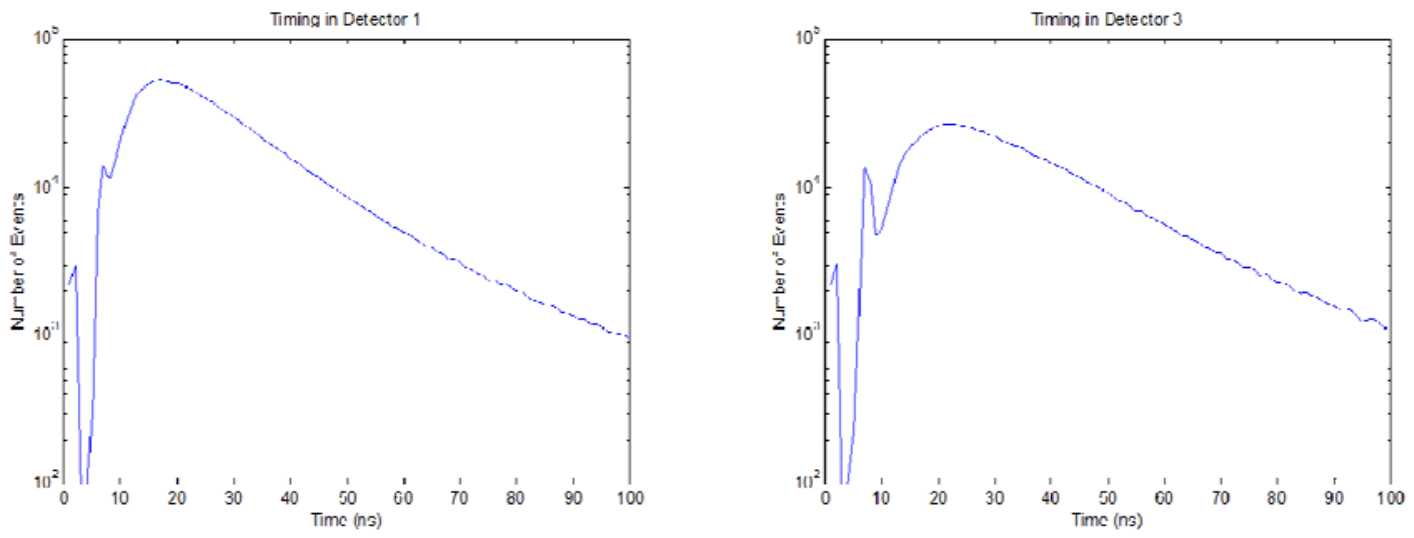

Fig. 14. Timing of the detection in the DU-polyethylene-DU configuration. 


\subsection{COMPUTER SIMULATION - EIGHT-DETECTOR SYSTEM}

Similar configurations were simulated with an eight-detector system. As in the four-detector system, initial analysis concerned the timing of events among the detectors. Fig. 15 shows the timing of events in detectors during the first $100 \mathrm{~ns}$ after a source emission for the top four detectors for the system depicted in Fig. 3. It can be seen that gammas from fission arrived at the detectors at about 16 ns after source emission. Fission neutrons then arrived at the detectors at between 20 and 25 ns, with a peak number of fission neutrons arriving at about $30 \mathrm{~ns}$.
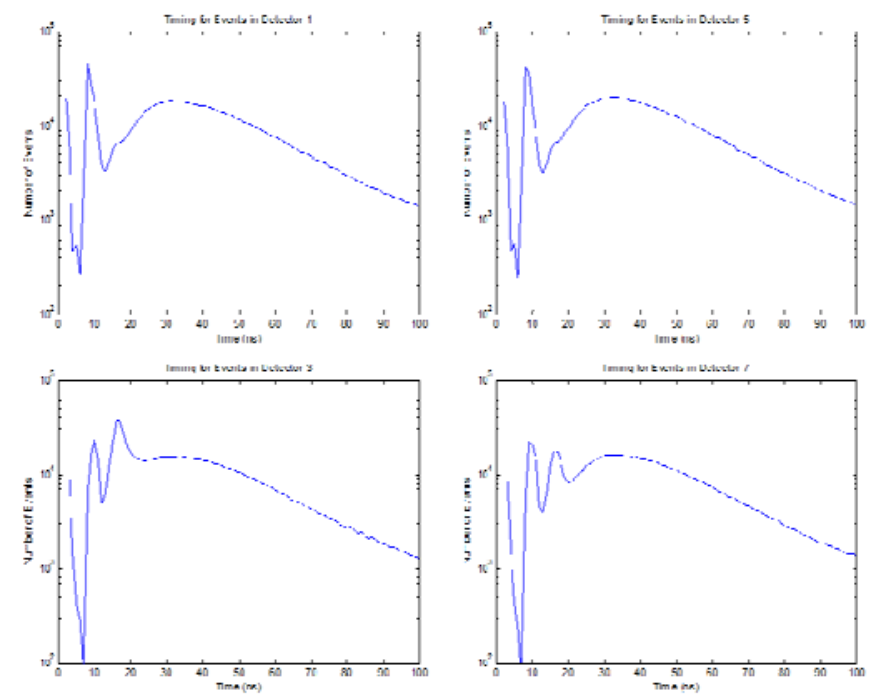

Fig. 15. Timing of events in the eight-detector system.

Fig. 16 shows the cross correlation in time of events occurring in pairs of detectors across the system. Gamma-gamma coincidences and gamma-neutron coincidences are more pronounced in these plots than in Fig. 7 because there was no lead shielding around the detectors. Looking at the half width at half maximum, it can be seen that events generally occurred in a second detector within 20 to 30 ns of a first event.
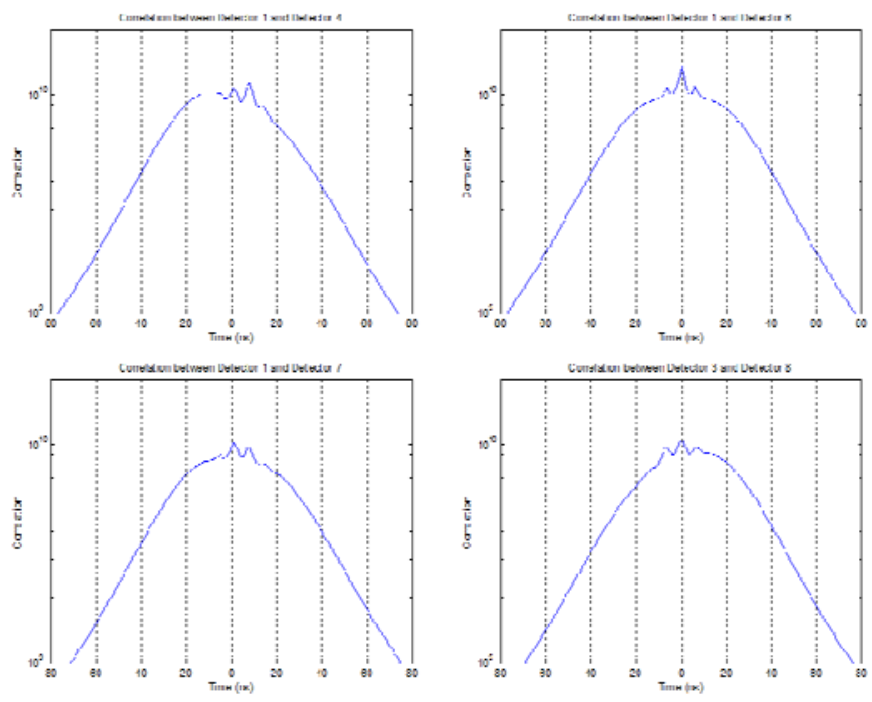

Fig. 16. Cross correlation of various detector pairs. 
To determine the occurrence of fissions associated with each pixel, the 25 to 40 ns time window, as well as other expanded time windows in which fission neutrons would probably be detected in the organic scintillators, were investigated . The expanded time windows were chosen because the timing plots (provided in Fig. 21) showed that detection of fission events occurred after about $20 \mathrm{~ns}$, and the cross correlation plots (shown in Fig. 22) indicated that events between detectors generally occurred within 20 to 30 ns of each other. Table 5 lists the time windows that were investigated for the fourdetector PoliMi simulation.

Table 5. Time windows

investigated for the eightdetector PoliMi simulation

\begin{tabular}{c}
\hline Investigated time windows \\
\hline 25 to $40 \mathrm{~ns}$ \\
25 to $45 \mathrm{~ns}$ \\
25 to $50 \mathrm{~ns}$ \\
30 to $40 \mathrm{~ns}$ \\
30 to $45 \mathrm{~ns}$ \\
30 to $50 \mathrm{~ns}$ \\
\hline
\end{tabular}

The Feynman variance by pixel for all of the time windows is shown in Fig. 17. Comparing the different time windows, it can be seen that the 25 to 50 ns time window provided the most evident differences in Feynman variance, so it was chosen as the time window to be used with subsequent simulations with different configurations. In this configuration, the neutron beams associated with pixels 05 and 13 were incident on the side of the annulus. Pixels 07, 09, and 11 had neutron beams centrally incident on the annulus. Pixels 03 and 15 were both partially incident on the lead shield around the DU target. As in the four-detector system, the centrally oriented neutron beams showed a lower Feynman variance than did the neutron beams oriented on the outer portions of the annulus. Pixels 03 and 15 also showed elevated Feynman variances due to the $(n, 2 n)$ multiplication in the lead shielding.
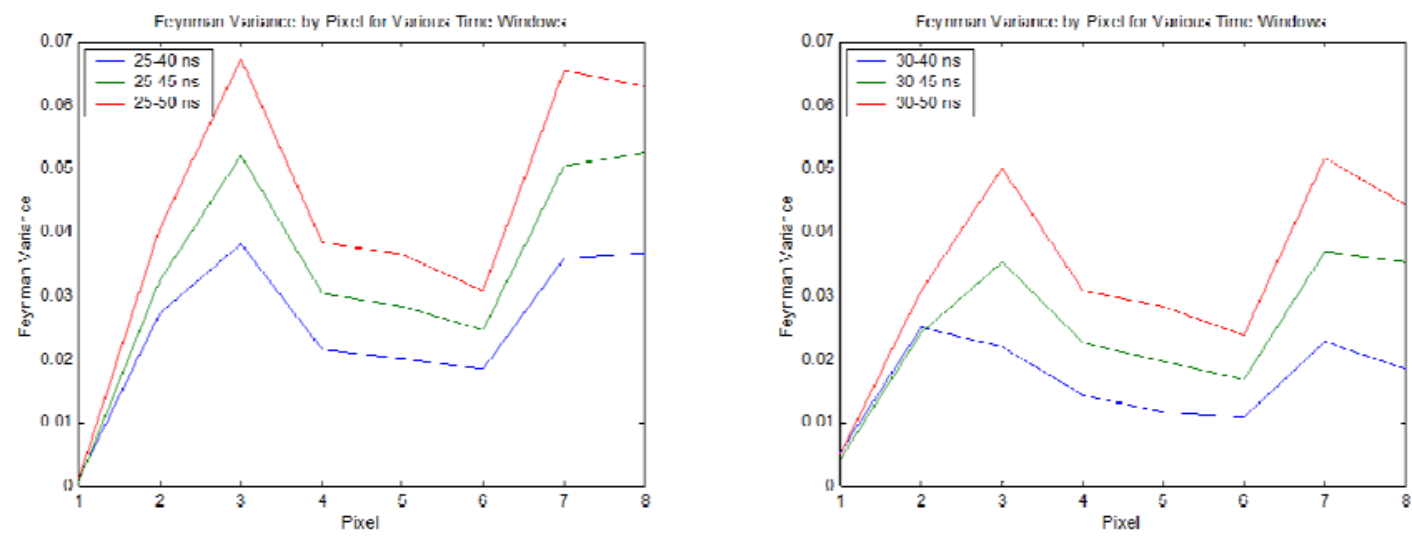

Fig. 17. Feynman variance by pixel for the eight-detector simulation for various time windows after source emission.

Interrogation of DU targets in several different configurations was also simulated with the PoliMi code. The first of these is depicted in Fig. 18, in which the DU annulus has been turned on its side with the side presented to the D-T generator. In this configuration, the pixel 01 neutron beam was not incident on the annulus. The pixel 03 and 15 neutron beams were each partially incident on the lead shielding. The pixel 05 and 13 neutron beams were incident on the ends of the annulus. The 
remaining neutron beams were all directly incident on the target. The Feynman variance plot in Fig. 18 shows elevated values for the pixels with associated neutron beams incident on the annulus, which is consistent with the expected results.
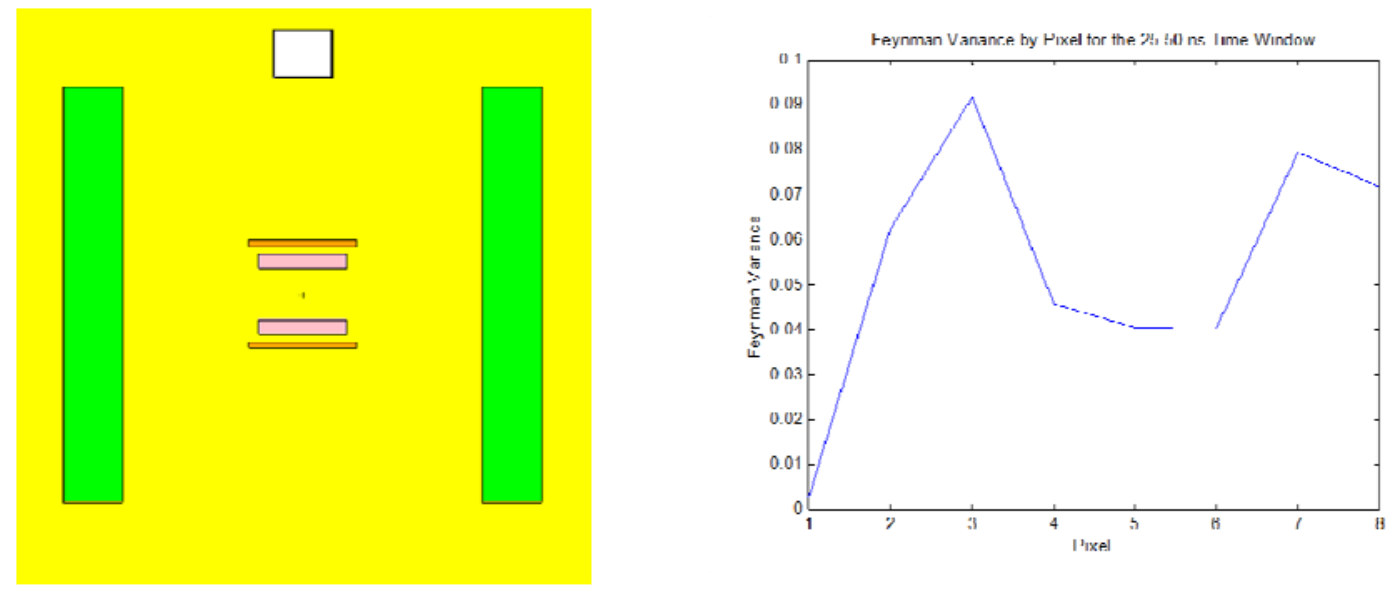

Fig. 18. PoliMi simulation results for DU annulus with side toward generator.

The next configuration to be simulated was the same DU annulus on its side with the opening presented to the neutron generator, depicted in Fig. 19. In this configuration, neutron beams associated with pixels $05,07,11$, and 13 were incident on the leading edge of the DU annulus. The pixel 03 and 15 neutron beams were incident on the leading edge of the lead shielding. Due to beam spreading, the beam associated with pixel 09was incident on the inside of the trailing edge of the DU annulus. The Feynman variance plot in Fig. 19 shows elevated values for the pixels with associated neutron beams incident on the annulus, which is consistent with the expected results.
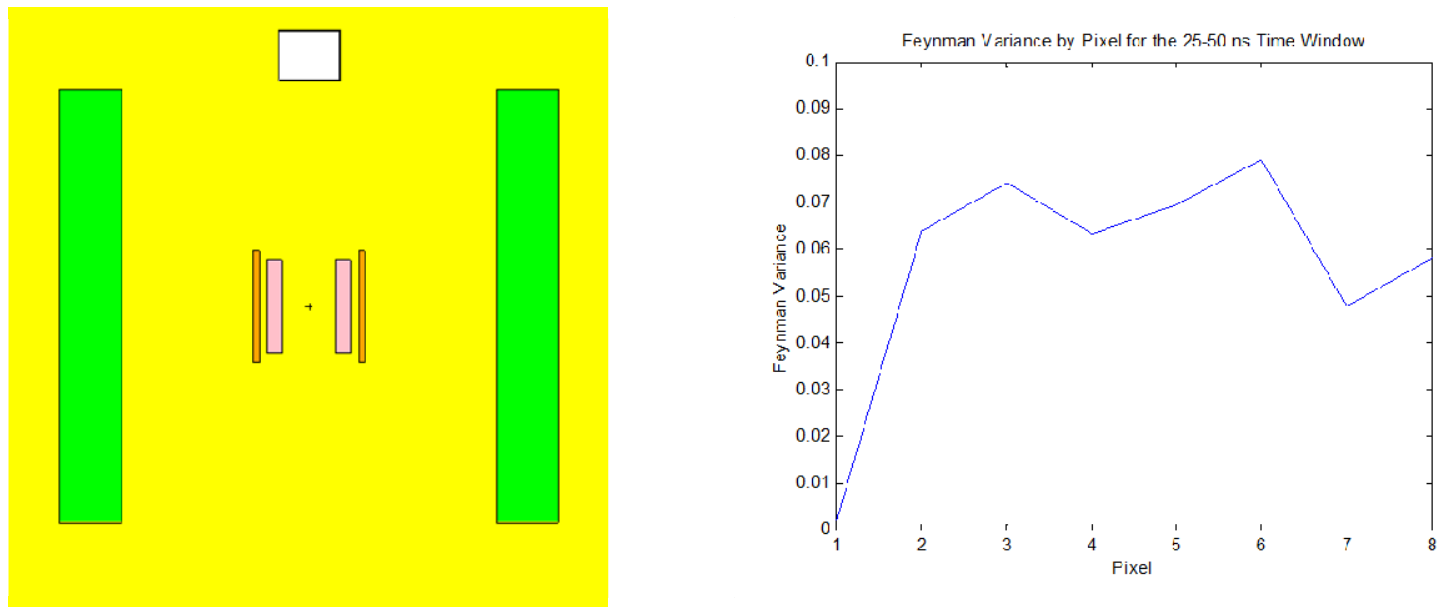

Fig. 19. PoliMi simulation results for DU annulus with opening toward generator.

The final configuration to be simulated with the eight-detector system had the same two DU annuli from the four-detector system separated by a $10 \mathrm{~cm}$ diameter cylinder of water, as depicted in Fig. 20. In this configuration, neutron beams associated with pixels 01, 03, 05, 13, and 15 were fully incident on the DU annuli. The pixel 07 and 11 neutron beams were incident on the water and the DU annuli. The remaining beam was fully incident on the cylinder of water. The fact that no multiplicity corresponding to fissions induced by pixel 09 neutrons scattered into the annuli from the cylinder of water was measured was unexpected; however, it is possible that there were few induced fissions due 
to scattered neutrons, and the particles generated by these fissions were attenuated by the targets. The Feynman variance plot in Fig. 20 shows a marked improvement of the eight-detector system over the four-detector one. First, because the size of the system was larger compared to the size of the target, fission particles were detected in a larger time window. Additionally, the larger number of detectors in each bank made it more likely that multiple detections of fission particles would be generated near one bank, even if there was self-attenuation in the target.
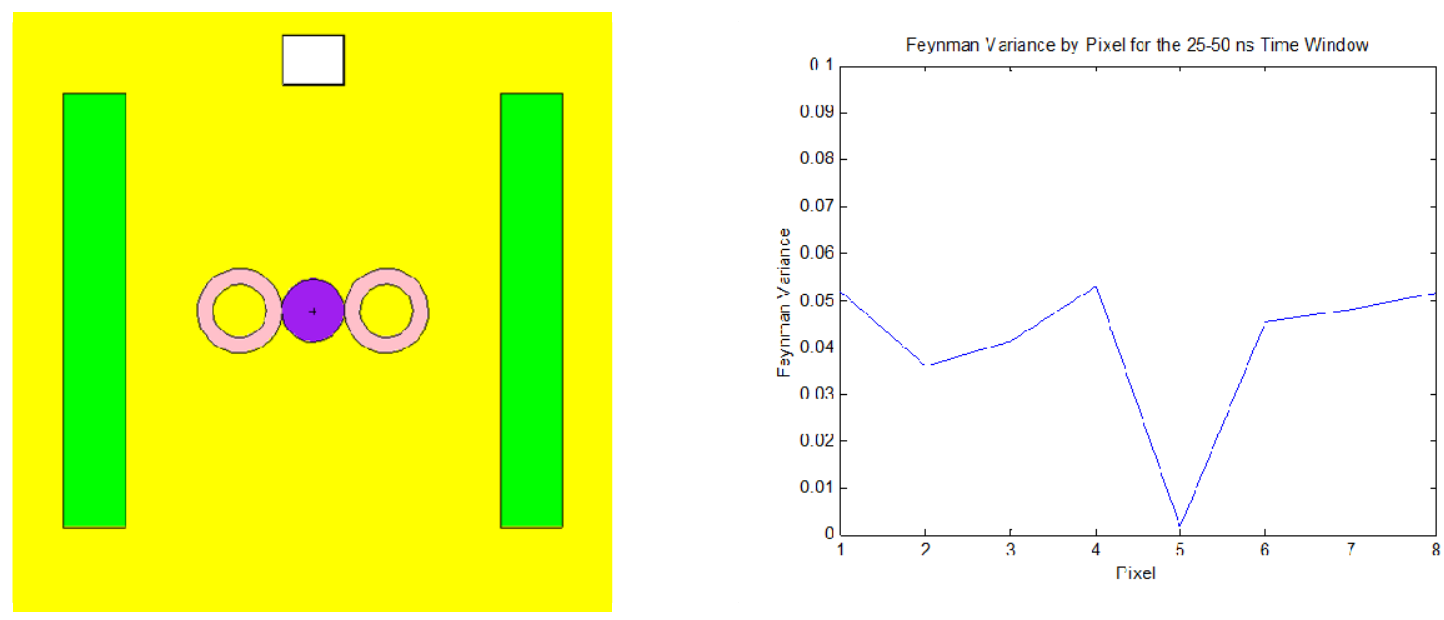

Fig. 20. PoliMi simulation results for two annuli separated by water.

\subsection{EXPERIMENTAL MEASUREMENTS - FOUR-DETECTOR SYSTEM}

Initial analysis of the experimental results concerned the timing of detection events among the four detectors. The previous timing calculations based on neutron velocity showed that neutrons should arrive at the various detectors in the 18 to 29 ns time window, which was used at the starting point for deciding the time windows to be investigated for this detector system. Fig. 21 shows the timing of events in detectors during the first $60 \mathrm{~ns}$ after a source emission. In this figure, the random detection events associated with the pixel have been subtracted out. It can be seen that gamma rays from fission began to be detected around 8 to 9 ns after source emission, and neutrons from fission began to be detected around 15 ns after source emission. Looking at the cross-correlation plots in Fig. 22, it can be seen that the half width at half maximum was generally 10 to $30 \mathrm{~ns}$. Therefore, based on the timing calculations and the timing and correlation plots, the time windows to be investigated were those listed in Table 6.

The Feynman variance by pixel for all of the time windows is shown in Fig. 23. Comparing the different time windows, it can be seen that the 18 to 40 ns time window provided the most evident differences in Feynman variance, while also maintaining Feynman variance values close to zero for those neutron beams that were not incident on the fissile target; therefore, it was chosen as the time window to be used with subsequent measurements with different configurations. In this configuration, the neutron beams associated with pixels 05 and 13 were partially incident on the side of the annulus. Pixels 07, 09, and 11 had neutron beams centrally incident on the annulus. As in the simulated system, the centrally oriented neutron beams showed a lower Feynman variance than did the neutron beams oriented on the outer portions of the annulus. 

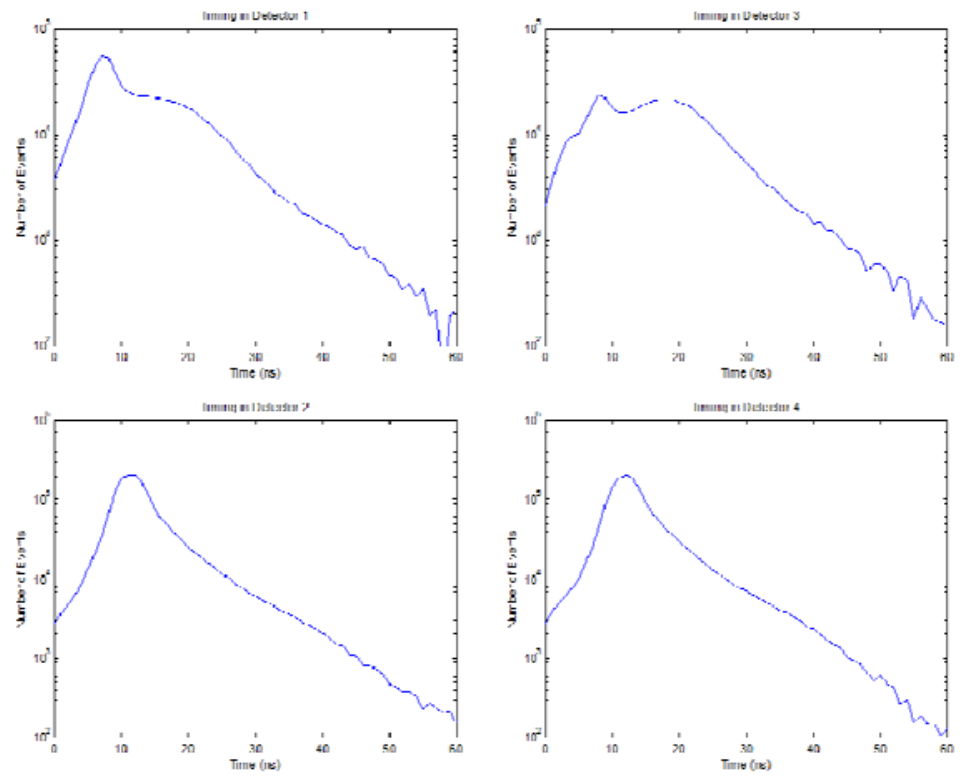

Fig. 21. Timing of detection events following source emission.
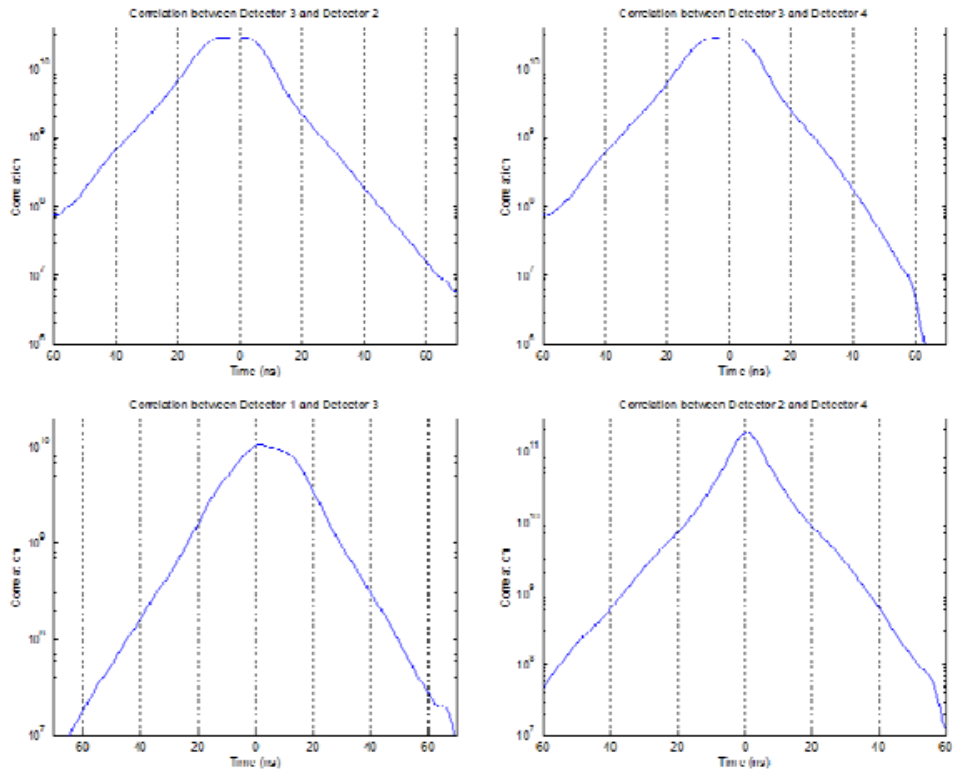

Fig. 22. Cross correlation of various detector pairs. 
Table 6. Time windows

investigated for the four-

detector experimental

measurements

\begin{tabular}{c}
\hline Investigated time windows \\
\hline 18 to $29 \mathrm{~ns}$ \\
18 to $35 \mathrm{~ns}$ \\
18 to $40 \mathrm{~ns}$ \\
18 to $45 \mathrm{~ns}$ \\
18 to $50 \mathrm{~ns}$ \\
25 to $35 \mathrm{~ns}$ \\
25 to $40 \mathrm{~ns}$ \\
25 to $45 \mathrm{~ns}$ \\
25 to $50 \mathrm{~ns}$ \\
\hline
\end{tabular}
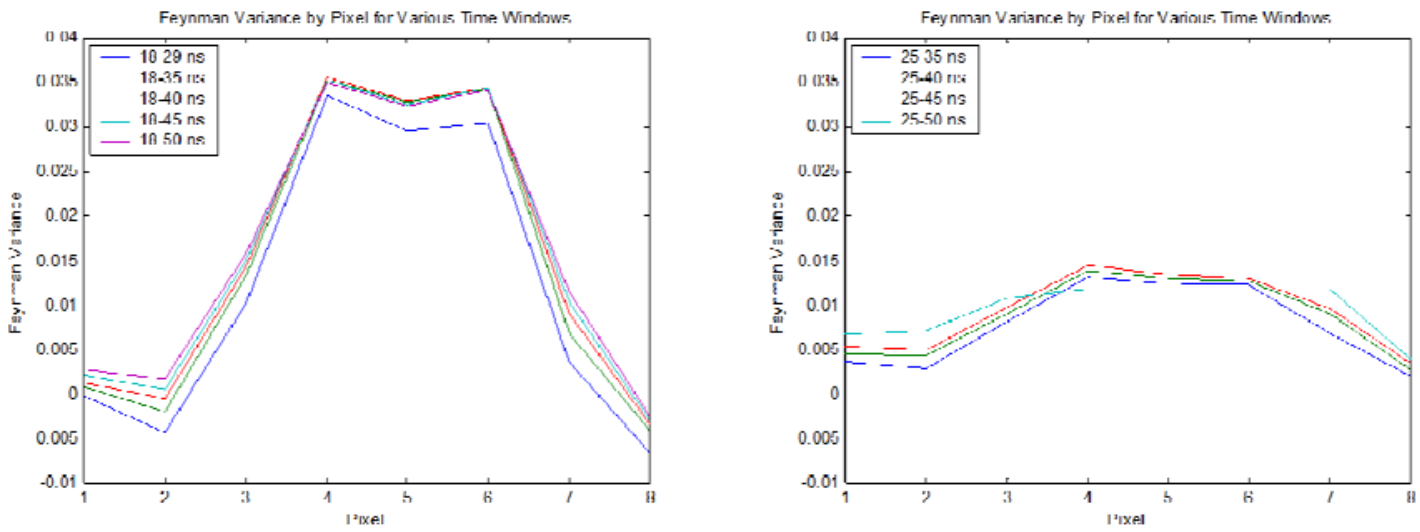

Fig. 23. Feynman variance by pixel from experimental results for various time windows after source emission.

A deviation of the experimental distribution of the number of counts occurring in the 18 to $29 \mathrm{~ns}$ time window from a Poisson distribution for neutron beams incident on the fissile material would be consistent with the results depicted in Fig. 23. Meanwhile, those beams that were not incident on the fissile material should show little or no deviation from the Poisson distribution. This is further illustrated in Fig. 24, in which the measured distribution of counts closely follows the Poisson distribution for the pixel 01 neutron beam. The pixel 07 neutron beam, being incident on the depleted uranium casting, caused a deviation of the correlated distribution of counts from the Poisson distribution.

It is interesting to note that although on average 4.4 neutrons are emitted from each fissioning nucleus, there are very few occurrences of more than three detections within the investigated time window depicted in Fig. 24. Because the fission neutrons are emitted isotropically, this is due to the limited amount of solid angle covered by the four neutron detectors. Additionally, because there is a 100 ns dead time for each detector, a maximum of only four detections can occur within the investigated time window. The average number of detections within the time window can be increased by increasing the number of detectors around the interrogated object. This approach would have the added benefit of enlarging the amount of solid angle covered by the detectors and would increase the probability of detecting more neutrons within the time window. 

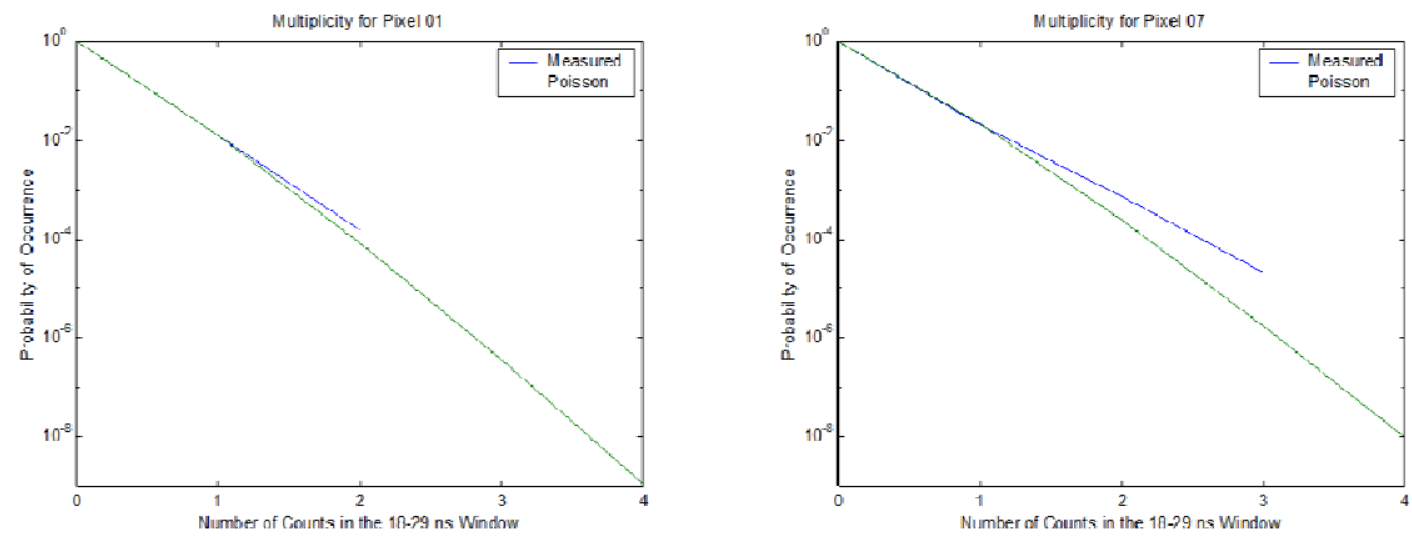

Fig. 24. Probability that $\boldsymbol{n}$ pulses occur in the 18 to 40 ns window for pixels 01 and 07.

DU targets in several different configurations were also interrogated for spatial multiplicity measurements. The first of these is depicted in Fig. 25, in which the DU annulus has been turned on its side with the side presented to the D-T generator. In this configuration, the pixel $01,03,05$, and 15 neutron beams were not incident on the annulus. The remaining neutron beams were all incident on the target. The Feynman variance plot in Fig. 25 shows elevated values for the pixels with associated neutron beams incident on the annulus, which is consistent with the expected results.
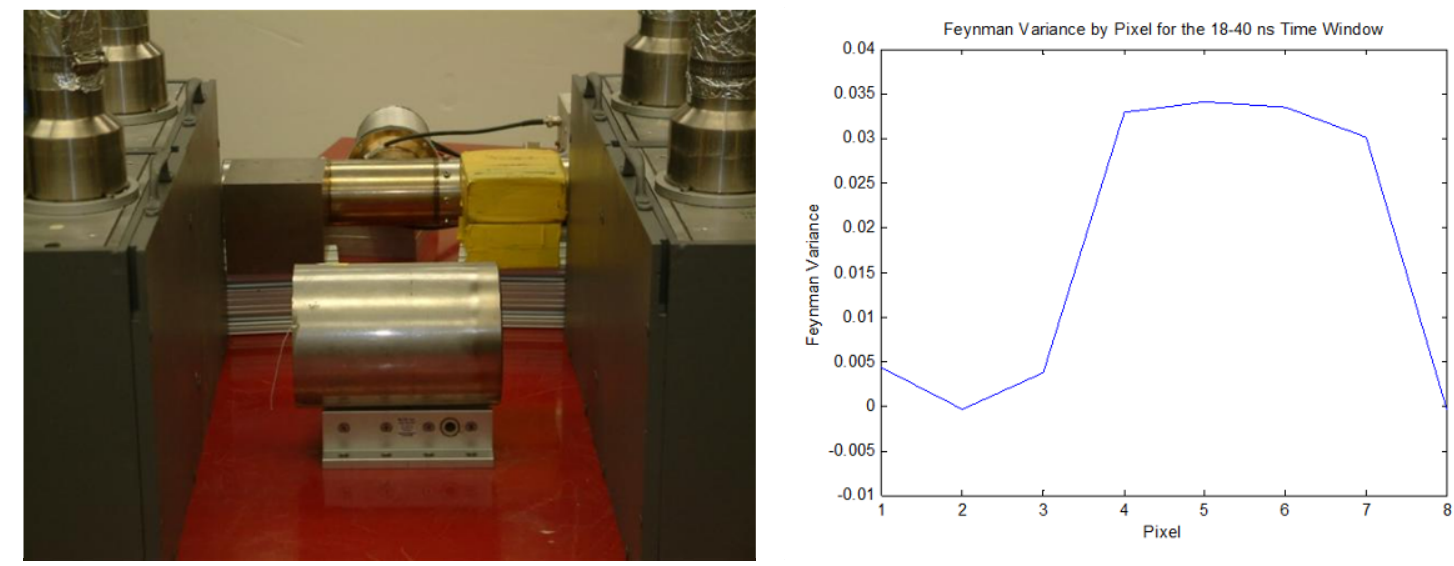

Fig. 25. Experimental results for DU annulus with side toward generator.

The next configuration to be interrogated was the same DU annulus on its side with the opening presented to the neutron generator, depicted in Fig. 26. In this configuration, neutron beams associated with pixels 07,11 , and 13 were incident on the leading edge of the annulus. Due to beam spreading, the beam associated with pixel 09 was incident on the inside of the trailing edge of the annulus. The Feynman variance plot in Fig. 26 shows elevated values for the pixels with associated neutron beams incident on the annulus, which is consistent with the expected results. 

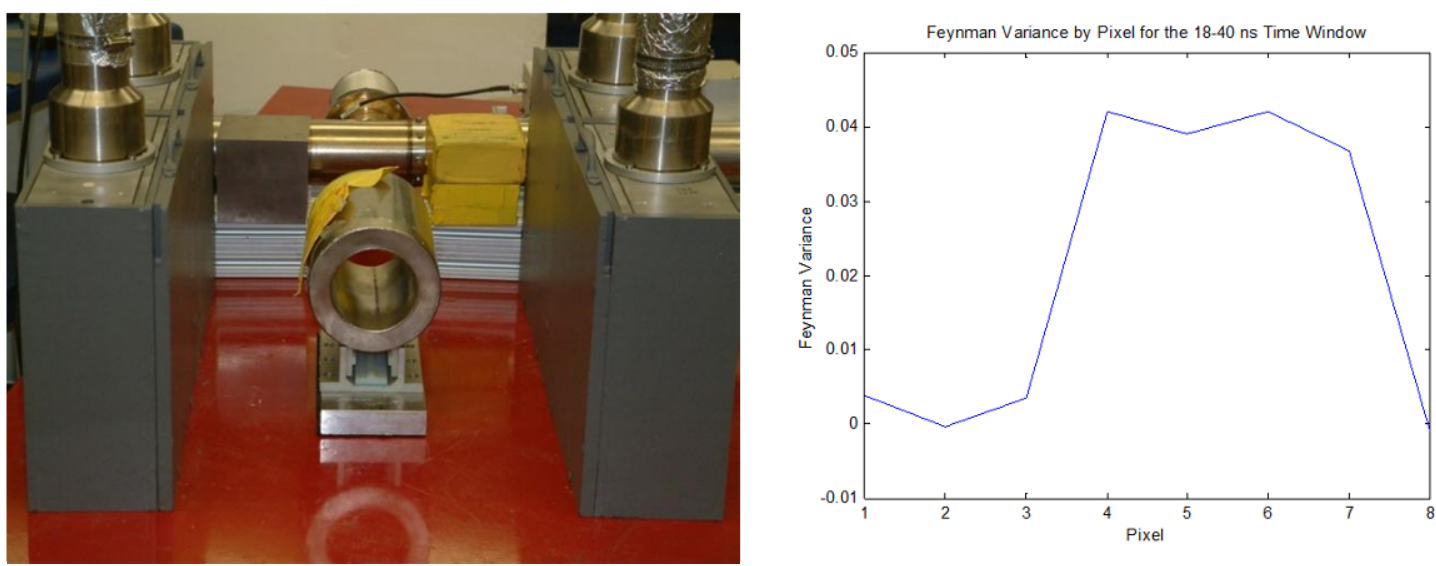

Fig. 26. Experimental results for DU annulus with opening toward generator.

The final configuration to be measured with the four-detector system had two DU annuli directly adjacent to the banks of detectors, as depicted in Fig. 27. Both annuli were identical, except the annulus on the right was half the height of the original annulus. The annuli were separated by a polyvinyl chloride cylinder filled with polyethylene beads. In this configuration, neutron beams associated with pixels $01,03,05,07,13$, and 15 were incident on the DU annuli. The remaining two neutron beams were at least partially incident on the polyethylene cylinder. The Feynman variance plot in Fig. 27 shows several characteristics about this interrogation method. First, the centrally oriented neutron beams that were incident on the polyethylene showed slightly elevated Feynman variance values, indicating that neutrons scattered off of the polyethylene-induced fissions in the annuli.
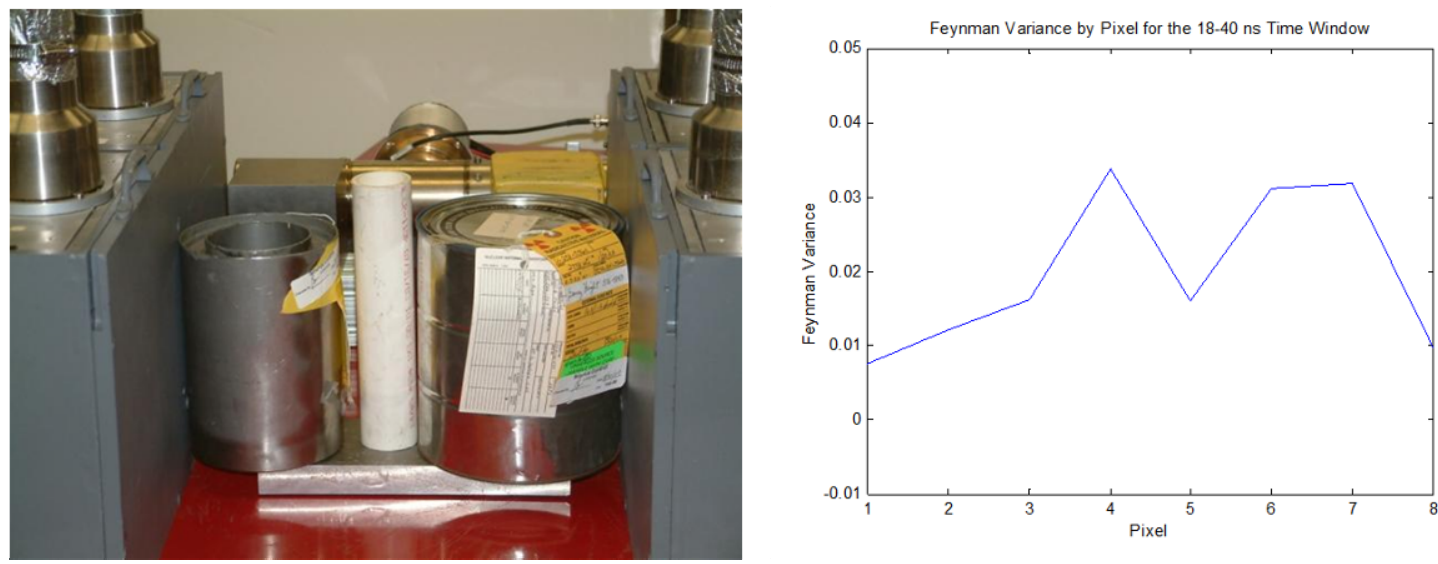

Fig. 27. PoliMi simulation results for two annuli separated by polyethylene.

Finally, the plot shows lower-than-expected Feynman variance values for the beams associated with pixels 01, 03, 05, and 15. This finding is similar to the effect observed in the PoliMi simulation of this target configuration and can once again be explained by the self-attenuation of the target and the geometric dependence of the investigated time window. Fig. 28 shows a skewing and broadening of the timing of detection events in this configuration similar to those seen in the PoliMi simulation. 

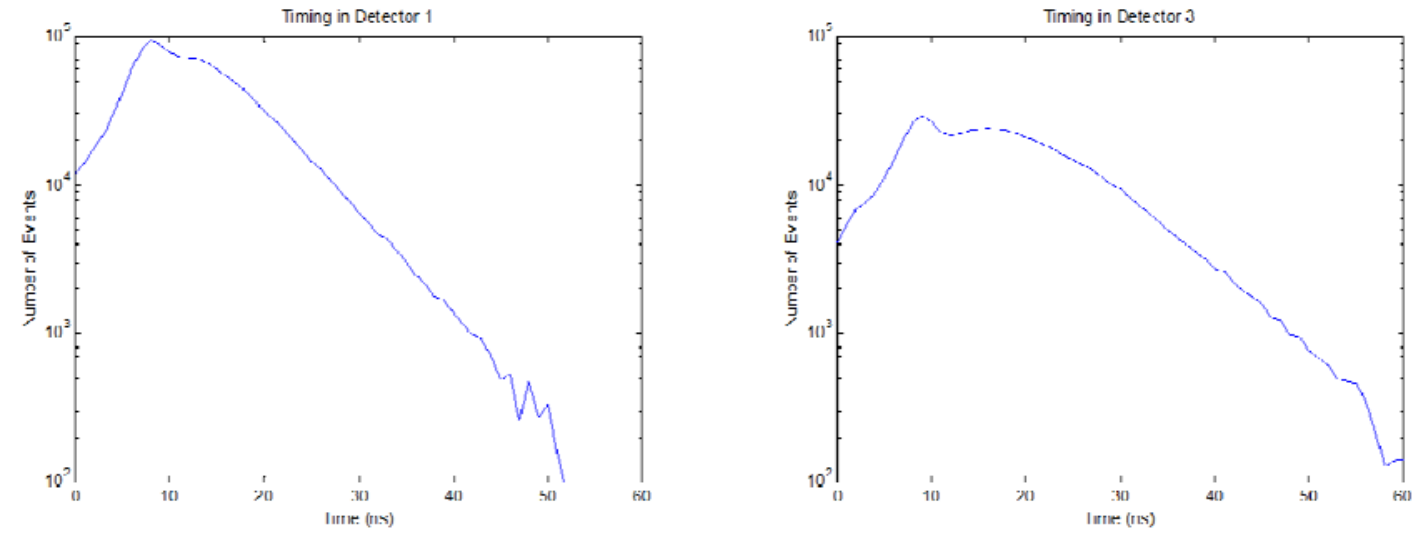

Fig. 28. Timing of the detection in the DU-polyethylene-DU configuration.

\subsection{MEASUREMENTS - EIGHT-DETECTOR SYSTEM}

Measurements were performed for similar configurations with an eight-detector system. As in the four-detector system, initial analysis concerned the timing of events among the detectors. Fig. 29 shows the timing of events in detectors during the first $100 \mathrm{~ns}$ after a source emission for the top four detectors for the system. It can be seen that gammas from fission arrived at the detectors at about 10 to $15 \mathrm{~ns}$ after source emission. Fission neutrons then arrived at the detectors beginning at $20 \mathrm{~ns}$, with a peak number of fission neutrons arriving at about $30 \mathrm{~ns}$.
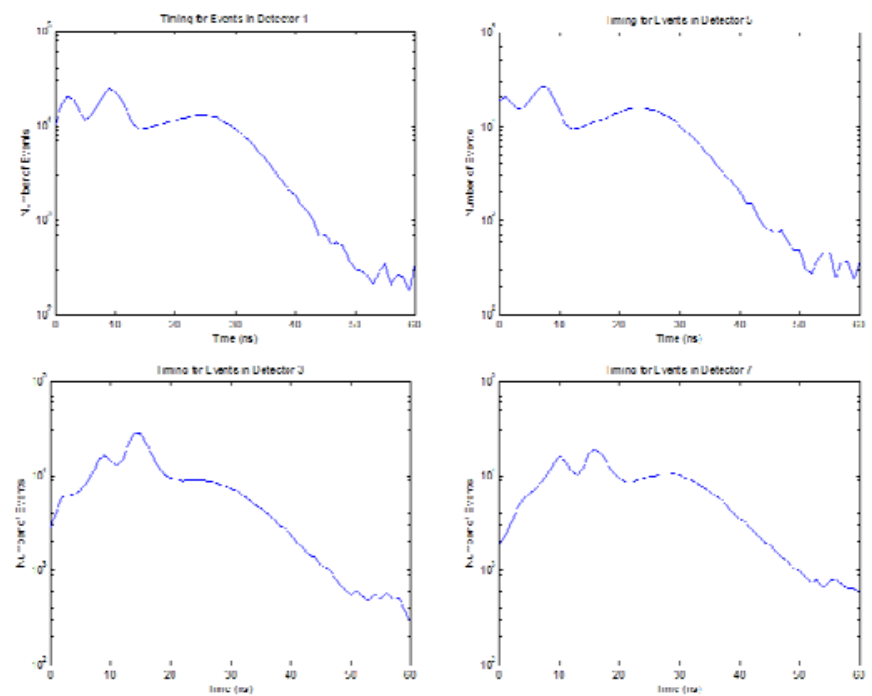

Fig. 29. Timing of events in the eight-detector system.

Fig. 30 shows the cross correlation in time of events occurring in pairs of detectors across the system. Looking at the half width at half maximum, it can be seen that events generally occurred in a second detector within 20 to 30 ns of a first event. 

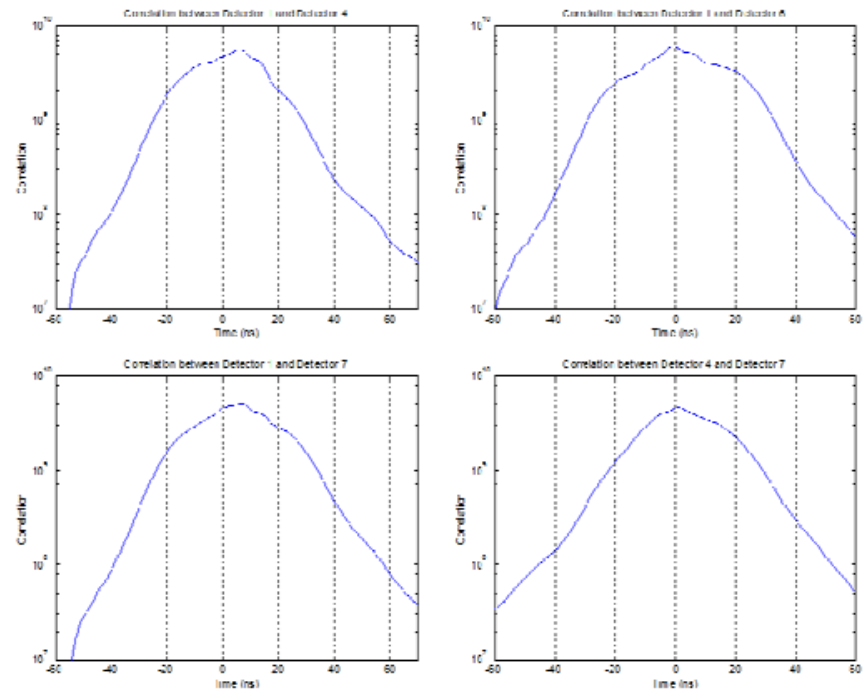

Fig. 30. Cross correlation of various detector pairs.

To determine the occurrence of fissions associated with each pixel, the 25 to 40 ns time-of-flight time window, as well as other expanded time windows in which fission neutrons would probably be detected in the organic scintillators, were investigated. The expanded time windows were chosen because the timing plots (provided in Fig. 29) showed that detection of fission events occurred after about 20 ns, and the cross-correlation plots (presented in Fig. 30) showed that events between detectors generally occurred within 20 to 30 ns of each other. Table 7 lists the time windows investigated for the eight-detector system.

Table 7. Time windows

investigated for the eightdetector experimental system

\begin{tabular}{c}
\hline Investigated time windows \\
\hline 20 to $35 \mathrm{~ns}$ \\
20 to $40 \mathrm{~ns}$ \\
20 to $45 \mathrm{~ns}$ \\
20 to $50 \mathrm{~ns}$ \\
25 to $40 \mathrm{~ns}$ \\
25 to $45 \mathrm{~ns}$ \\
25 to $50 \mathrm{~ns}$ \\
30 to $40 \mathrm{~ns}$ \\
30 to $45 \mathrm{~ns}$ \\
30 to $50 \mathrm{~ns}$ \\
\hline
\end{tabular}

The Feynman variance by pixel for all of the time windows is shown in Fig. 31. Comparing the different time windows, it can be seen that the 20 to 40 ns time window provided the most evident differences in Feynman variance while maintaining low Feynman variance values for the pixels without incident neutron beams. Therefore, it was chosen as the time window to be used in subsequent simulations with different configurations. In this configuration, the neutron beams associated with pixels 05 and 13 were incident on the side of the annulus. Pixels 07, 09, and 11 had neutron beams centrally incident on the annulus. Pixels 03 and 15 were both partially incident on the lead shield around the DU target. As in the four-detector system, the centrally oriented neutron beams showed a lower Feynman variance than did the neutron beams oriented on the outer portions of the 

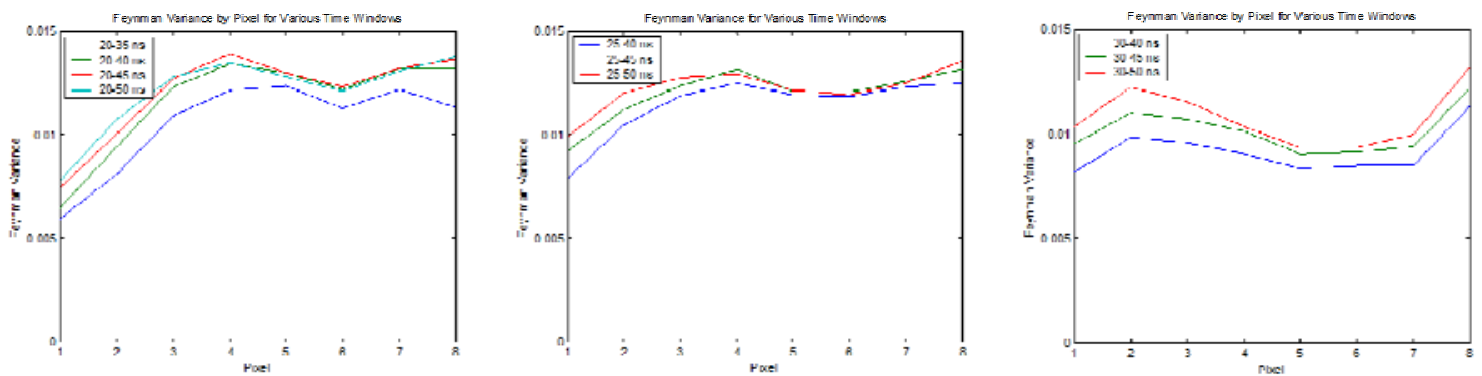

Fig. 31. Feynman variance by pixel for the eight-detector measurements for various time windows after source emission.

annulus. Pixels 03 and 15 also showed elevated Feynman variances due to the $(n, 2 n)$ multiplication in the lead shielding.

It is interesting to note that although the pixel-to-pixel Feynman variance differences were not as marked as for the four-detector configuration, there was an improvement in the ability to detect triple and quadruple coincidences. Fig. 32 shows the increased detection of triple and quadruple coincidences correlated to pixel 01, which did not have source neutrons incident on the target, and pixel 07, which did. Additionally, the Feynman variance values for the outer pixels were significantly higher than those for the eight-detector simulation. This deviation of the outer pixels from the Poisson distribution can be explained by the presence of materials in the environment that were not present in the simulation. A method for reducing environmental effects is to subtract a background measurement of the system without a target present from measurements with targets.
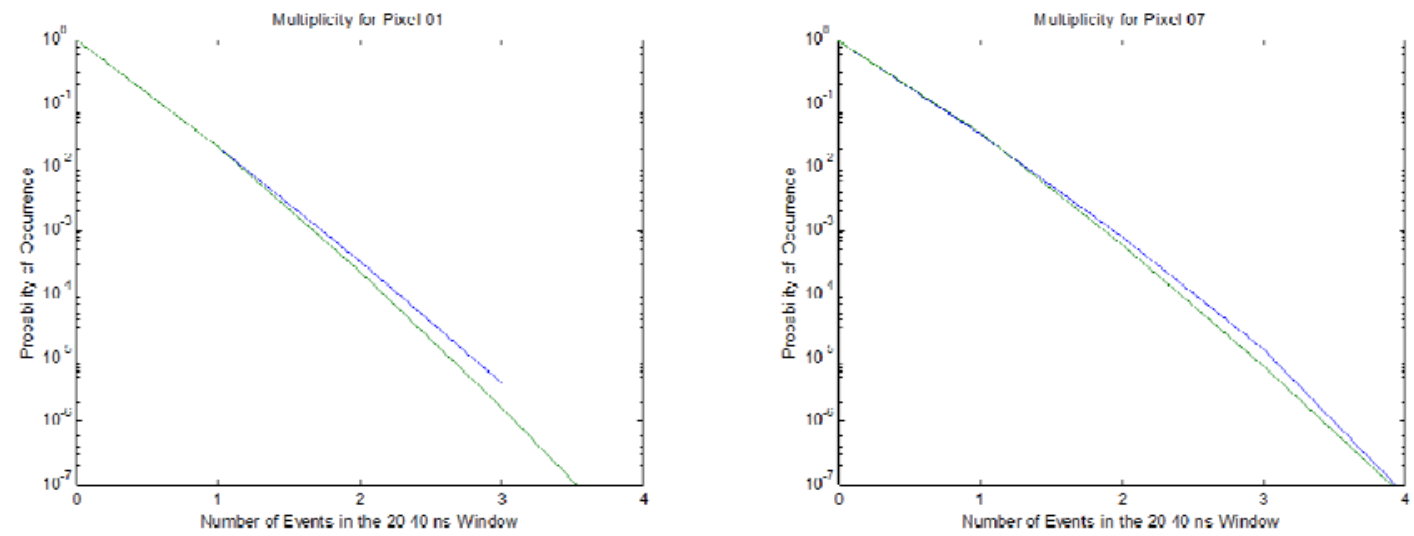

Fig. 32. Probability that $n$ pulses occur in the 20 to $40 \mathrm{~ns}$ window for pixels 01 and 07.

\section{CONCLUSIONS}

Detecting multiple neutrons in a set time window after a neutron-generation event can indicate that there is fissile material present in an interrogated object. The Feynman variance is the value used to measure the multiple detections. The optimum time window for counting multiple detections associated with nonrandom phenomena such as fissions can be estimated and will be based on the geometry of the system. It has been shown that the Feynman variance of detection events that occur within a set time window can be correlated to a specific neutron beam. Because each beam is 
associated with a direction of emission from the neutron generator, this information can give an indication of the spatial distribution of fissile material in the interrogated object.

It has been shown that increasing the number of detectors surrounding the target increases the probability of detecting double, triple, and quadruple coincidences; however, it also increases the effects of multiplying materials in the environment. Future work should investigate the effects of subtracting a background measurement from target-interrogation measurements. An additional area for future investigation is to correlate the spatially distributed Feynman variance values with the spatially distributed fission density of the target.

\section{REFERENCES}

1. D.L. Chichester, M. Lemchak, and J.D. Simpson, "The API 120: A Portable Neutron Generator for the Associated Particle Technique,” Nuclear Instruments and Methods in Physics Research B 241, 753-8 (2005).

2. P.A. Hausladen, J.S. Neal, and J.T. Mihalczo, “An Alpha Particle Detector for a Portable Neutron Generator for the Nuclear Materials Identification System (NMIS),” Nuclear Instruments and Methods in Physics Research B 241, 835-8 (2005).

3. J. T. Mihalczo et al., "Physical Description of Nuclear Materials Identification System (NMIS) Signatures,” Nuclear Instruments and Methods in Physics Research A 450, Nos. 2-3, 531-55 (August 2000).

4. T. Ethvignot et al., "Neutron Multiplicity in the Fission of ${ }^{238} \mathrm{U}$ and ${ }^{235} \mathrm{U}$ with Neutrons up to 200 MeV,” Physical Review Letters 94, 052701/1-4 (2005).

5. J.A. Mullens et al., "Fast Coincidence Counting with Active Inspection Systems," Nuclear Instruments and Methods in Physics Research B 241, 804-9 (2005).

6. R.P. Feynman, F. de Hoffman, and R. Serber, "Dispersion of the Neutron Emission in U-235 Fission,” Journal of Nuclear Energy 3, 64-9 (1956).

7. T. Ethvignot et al., "Prompt-Fission-Neutron Average Energy for ${ }^{238} \mathrm{U}(\mathrm{n}, \mathrm{f})$ from Threshold to 200 MeV,” Physics Letters B 575, 221-8 (2003).

8. G.S. Brunson, "An Extension to the Feynman Method of Detecting Fission Neutrons," Nuclear Instruments and Methods 214, 341-8 (1983).

9. Y. Kitamura et al., "Feynman-Alpha Experiment with Stationary Multiple Emission Sources," Progress in Nuclear Energy 48, 569-77 (2006).

10. P. Hausladen, personal communication to J. Mihalczo, October 2007.

11. E. Padovani and S.A. Pozzi, MCNP-PoliMi User's Manual, Version 1.0, Milan, Polytechnic of Milan, November 2002. 